\title{
Secado de colas filtradas en contacto con la atmósfera
}

\author{
Germán Rodari y Luciano A. Oldecop. \\ Instituto de Investigaciones Antisísmicas “Ing. Aldo Bruschi", Universidad Nacional de San Juan (UNSJ), Av. Libertador \\ General San Martín 1290 Oeste. \\ germanrodari@gmail.com; luciano.oldecop@gmail.com
}

\begin{abstract}
RESUMEN
La tecnología de colas filtradas permite separar y reutilizar parte del agua de los residuos antes de su disposición. Las colas filtradas se almacenan formando una pila sin necesidad de construir una presa. El desempeño de este tipo de depósitos depende del grado de saturación que alcancen las colas al momento del cierre. En este trabajo se propone un modelo conceptual que describe los procesos y variables determinantes de la evolución de la humedad y del grado de saturación durante la construcción. Se analizan datos obtenidos en la mina Casposo (San Juan, Argentina) para estudiar, por un lado, el proceso de secado mientras las colas están expuestas a la atmósfera y por otro lado el proceso de consolidación bajo la carga impuesta por las tongadas subsiguientes. El concepto de humedad objetivo al momento de cubrir las colas con la siguiente capa, sólo resulta útil si se tiene en cuenta su variabilidad. Ésta se puede caracterizar, para el caso estudiado, como una variable aleatoria normalmente distribuida. Por otra parte, se define el concepto de humedad de saturación, como el valor que causará la saturación de las colas cuando se sometan a la tensión vertical máxima.También ésta resulta una variable aleatoria normalmente distribuida. Comparando ambas variables es posible calcular la probabilidad de alcanzar la saturación en una capa ubicada a una cierta profundidad. Esto permite evaluar si el grado de saturación al finalizar la construcción estará dentro de lo deseable. Además, proporciona un procedimiento para analizar y ajustar el diseño.
\end{abstract}

Palabras clave: colas filtradas, depósito, geotecnia, humedad, minería.

\section{Drying of filtered tailings in contact with the atmosphere}

\begin{abstract}
Filtered tailings technology enables the separation and reuse of part of the water from solid waste, before its disposal. The filtered tailings can be piled in a stack, avoiding the need for a dam. The behavior of such deposits depends on the degree of saturation of the tailings at the closure stage. This paper proposes a conceptual model describing the processes and variables determining the evolution of the water content and the degree of saturation throughout the construction of the deposit. We have obtained data from the Casposo mine (San Juan, Argentina) and this has been analysed to study, on the one hand the drying process while the tailings remain exposed to the atmosphere and, on the other hand, the consolidation process under the load imposed by subsequent layers. The concept of target water content at the moment of covering the tailings under the next layers is only useful if its variability is taken into account. This can be characterized, for the case studied, as a normally distributed random variable. The concept of saturation water content is defined as the value that will cause saturation of the tailings when subjected to the maximum vertical stress. This is also a normally distributed variable. By comparing both variables, the probability of reaching saturation in a layer located at a certain depth is computed. This allows us to evaluate if the degree of saturation by the end of the construction will be within the desirable range. It also provides a tool to analyse and adjust the design.
\end{abstract}

Keywords: deposit; filtered tailings; geotechnics; mining; water content. 


\section{Introduction}

Filtered tailings are a relatively new mine waste disposal technique which has multiple advantages: to avoid the construction of a dam, to minimize contaminating leakages and to save water, for instance. Filtered tailings can be stacked in layers, forming a pile. The key for the success of such a deposit is to achieve and maintain the tailings in a permanent unsaturated state. The recommended saturation degrees range from 70 to $85 \%$ (Davies and Rice, 2001). Values near the upper end of this range or slightly above are preferable to prevent acid drainage (Ulrich and Coffin, 2013).

Figure 1 shows a conceptual model, describing the main processes undergone by the tailings after their output from the extraction process. Four stages are identified. Stage 1 includes filtration, in which the water content value is lowered to $w_{f}$, with typical values ranging from 20 to 45\% (Wang et al., 2014). Stage 2 initiates when the tailings are discharged in the deposit. Several physical processes take place, including evaporation, rain, freezing and melting, infiltration, runoff and eventually earthwork operations, such as grading, ploughing to accelerate drying (Caldwell and Crystal, 2015) or compaction (Lara et al., 2013). As a result, the water content may increase or decrease. Stage 2 ends when the tailings are buried under a new construction layer and the water content is $w_{0}$. During stage 3 , the water content is expected to remain nearly constant, since evaporation is limited to a shallow active zone (Garino and Oldecop in this volume). On the other hand, the saturation degree will increase from an initial value $S_{0}$ (at placement) to final value $S_{c}$ as the void volume reduces, due to the overburden stress increasing during construction. Stage 4 starts at the closure of the deposit. If an adequate cover system is provided, the final saturation degree $S$ should no longer change. By selecting a suitable value for $w_{0^{\prime}}$ it is possible to ensure that by the end of stage 3 and in stage 4 an adequate degree of saturation, $S$, will be attained. Such a value depends on compressibility of the tailings and on the overburden stress at the end of Stage 3.

This paper analyses field and lab data obtained at the Casposo mine (San Juan, Argentina), in order to evaluate the applicability of the conceptual model proposed for filtered tailings deposits. At the Casposo mine, located in the central Andes of Argentina, the tailings are filtered by vacuum application on conveyor belts (Fig. 2). The filtered tailings are then transported in trucks to the deposit, where they are spread in layers (Fig. 3). The deposit consists of layers of tailings alternating with waste rock layers (Fig. 4), both $1 \mathrm{~m}$ thick, placed with no compaction.

\section{Drying of filtered tailings exposed to the atmosphere}

Two different placing procedures have been studied: dumping without further handling and dumping followed by spreading and ploughing.
The drying of $1 \mathrm{~m}$ thick tailings layers placed by dumping with no further handling was investigated by collecting samples to measure the water content. Samples were taken from the surface and at a $0.50 \mathrm{~m}$ depth within the upper tailings layers, while in contact with the atmosphere (stage 2), at different times elapsed from the discharge. The data are plotted in Figure 5 .a as gravimetric water content $(w)$ and also as water mass fraction $\left(w^{\prime}\right)$, as defined in equations (1) and (2). The two experimental datasets were fitted with equation (3), in which the first two terms represent the mean water content, , at time t. $\epsilon_{w}$ is the residual, assumed to be a normally distributed random variable, with zero mean and standard deviation, . Figure $5 b$ displays the variance analysis of $\epsilon_{w}$. which was observed to decrease as time elapsed and its variation is described by means of equation (4). The parameters of equations (3) and (4), obtained with least squares regression, are given in Table 2. Figure 5.a displays the mean value curve and the $90 \%$ confidence intervals obtained with equations (3) and (4). They suggest that after a certain time the drying process stops. Figure 6 presents a series of moisture profiles and dry unit weight values obtained in trenches dug in layers of tailings in stage 2, at different times elapsed since placement. These data show the same trend, suggesting that the main cause of the drying stopping is the development of a shallow dry layer interrupting the capillary rise.

An alternative placing method was tried, with the aim of speeding-up the drying process. The daily production of the tailings was spread in a $0.40 \mathrm{~m}$ thick layer forming a parcel called a "cell." Each cell was moved daily with a disc plough (Fig. 7). The water content of the cells was measured daily in samples taken 20 $\mathrm{cm}$ below the surface. The data obtained are shown in Figure 8a along with the mean water content curve obtained by least squares fitting of equation (3) and the $90 \%$ confidence limit curves for the standard deviation obtained from the variance analysis shown in Figure $8 b$.

Figure 9 compares the performance of the two placing methods tested. For instance, at $t=45$ days the mean water content of tailings dumping without ploughing was $w=23 \%\left(w^{\prime}=18.7 \%\right)$. The same mean water content is reached for $t=10$ days in the case of tailings spread and ploughed.

\section{Tailings compressibility}

The compressibility of the tailings has been investigated through a series of oedometer tests. The tested materials are identified in Table 3 and the grain size distribution curves are shown in Figure 10. The 11 material consists in tailings sampled at the outlet of the Casposo processing plant, while the 12 material consists in mine ore, ground in a pilot plant, ahead of mine commissioning. The tested specimens were prepared with different initial water content, without 
compaction. The initial and final test conditions are summarized in Table 4. The initial void ratio was found to be water content dependent. In material 11 , the lower initial water content led to lower void ratios. In material t2 the trend was the opposite. This was attributed to particle aggregation due to the effect of the flocculant agent contained in 11 (Figs. 11a and 11b).

Figure 12.a shows the compression curves obtained in the oedometer tests and Figure $12 b$ displays the evolution of the saturation degree. In Figure 13a, the same experimental data are presented as the specimen water content vs. the applied vertical stress.

Field data points obtained from exploration pits are also included in Figure 13a as black circles. In this case, the saturation water content was computed from in situ unit weight (ASTM D1556) and the specific gravity determined in the lab (ASTM D854-14), whilst the vertical stress was estimated from the cover thickness and the average unit weight. The field data were fitted with equation (5) by means of least squares regression. The first two terms of Equation (5) yield the mean saturation water content value, , under a given vertical stress, where . is the residual, which is assumed as a normally distributed random variable with zero mean and standard deviation, , determined from the variance analysis shown in Figure 13b. Figure 13a displays the mean saturation water content line and the $90 \%$ confidence intervals obtained with equation (5) and the parameters specified in Table 5. The analysis presented is limited to tailings placed with no further handling. There are no data so far for the method with ploughing.

\section{Discussion}

By comparing the water content achieved after the $d r$ ying process, , with the saturation water content, , the saturation degree at closure stage, , can be estimated, thus allowing the selection of the tailings placing method.

The experimental data presented herein show that, given the duration, $t$, of the drying period and the vertical stress, acting in a layer at a certain depth, and can be described as normally distributed random variables. Moreover, if they are assumed to be independent, the probability of, which is the same as the probability of reaching saturation by the end of construction, can be computed by means of equation (6). Equation (6) can be extended to any saturation degree by replacing wsat with S.wsat. Moreover, by assuming mean values of dry unit weight and water content of the overburden material (for the present analysis $14.6 \mathrm{kN} / \mathrm{m} 3$ and $23 \%$, respectively), the vertical stress can be readily converted to depth-of-cover, $T$. Hence, the probability of to exceed a given value $S$ in a tailings layer having a water content and located at depth $T$ can be computed by means of equation (7), where $X$ is also a random variable having a mean value and standard deviation.
In order to highlight the potential practical applications of the proposed conceptual model, Figure 14 depicts the exceedance probability of the saturation degree at closure (Eq. 7) for a tailings layer having undergone a drying period of 45 days with no ploughing (Fig. 9), for different cover depths. The curves shown were computed by means of equations (3), (4), (5) and (7).

The results in Figure 14 show that saturation will be attained at some points of the deposit. For instance, in layer located at $20 \mathrm{~m}$ depth, $15 \%$ of its volume is expected to reach saturation. Due to the layered design of the deposit this does not present a problem. The free-draining waste rock layers guarantee that no excess pore pressure will develop. Moreover, considering the capillary barrier effect (Oldecop and Rodari, 2017), it can be expected that most of the water in excess will redistribute within the same layer by lateral flow. Evidence of lateral flow occurring in the deposit was inferred from the striped pattern observed in the deposit slopes (Fig. 15) arising from saline precipitations induced by the evaporation of tailings water to the atmosphere. In the long term, the saturation degree should evolve trending to a uniform saturation degree within each layer approaching the $50 \%$ probability values displayed by the curves of Figure 14.

\section{Conclusions}

Though this work was based on data from a particular case, the proposed conceptual model is intended as a basic framework for the design and operation planning of filtered tailings deposits.

The variability displayed by the field and lab data describing both the compressibility and drying process of the filtered tailings suggests that it is meaningless to attempt to set a single target water content value as design parameter. Certainly it has much more sense to include such variability in the analysis by means of suitable statistical tools.

Some considerations can be made about the design of this type of deposit. The drying time required in stage 2 geometrically constraints the deposit as it determines the work area necessary to accommodate the tailings production. The layered design of the case studied favours the redistribution of excess water content and eventually allows drainage, thus avoiding the build-up of excess pore water pressure. Given the lack of published information, the design of filtered tailings deposits must rely on test data, which may differ notably from the behaviour under real operating conditions. Hence, at the project stage, designers should foresee wide safety margins in the operation variables, allowing operating areas larger than strictly needed, for instance. Once in operation, the design and operational plan will almost certainly require updates, based on real data acquired during operation. 


\section{Introducción}

Las colas (del inglés tailings) o relaves, son un residuo de la minería compuesto por una mezcla de roca finamente molida, agua y aditivos. Separar y reutilizar parte del agua de las colas, antes de disposición final, presenta múltiples ventajas. Entre las tecnologías disponibles, el filtrado es la que mayor reducción de contenido de agua permite lograr. La consistencia de las colas filtradas hace imposible su transporte por tubería o canales, por lo que deben ser transportadas y colocadas en el depósito por medios mecánicos, tales como cintas transportadoras, camiones, topadoras, etc. Por otro lado, las colas filtradas poseen una consistencia suficiente como para sostenerse sin necesidad de una estructura de contención, como una presa. Los depósitos de colas filtradas se construyen disponiendo las colas en tongadas, de manera similar a la que se utiliza para formar terraplenes o pilas.

Además de evitar la construcción de una presa, la disposición de las colas filtradas tiene otras ventajas. El aprovechamiento del agua se optimiza al reducirse las pérdidas por evaporación y la cantidad de agua que queda almacenada en los poros. El menor contenido de agua en el residuo reduce el riesgo de filtraciones contaminantes superficiales o subterráneas. Otra ventaja es la posibilidad de llevar a cabo el plan de cierre paulatinamente, simultáneamente con el recrecimiento del depósito. Finalmente, las colas en estado no saturado tienen un mejor comportamiento frente a acciones sísmicas (Oldecop and Rodari, 2017). El objetivo de diseño de un depósito de colas filtradas es lograr que el grado de saturación final de las colas almacenadas esté por debajo del $100 \%$, con un cierto margen de seguridad. Sin embargo, no es conveniente un grado de saturación demasiado bajo ya que eso permite la difusión de oxígeno en las colas y con ello aumentaría el potencial de generar drenaje ácido. Los valores recomendados de grado de saturación van desde el 70 al 85\% (Davies and Rice, 2001) o algo superiores al $85 \%$ cuando se intenta prevenir el drenaje ácido (Ulrich and Coffin, 2013).

El filtrado se realiza con un tejido sintético, ya sea por aplicación de presión, vacío o aceleración en centrífuga. La eficiencia de la filtración, medida como el caudal de líquido separado por tonelada de sólidos procesados, crece con el área del filtro y con la diferencia de presión aplicada, mientras que decrece con la viscosidad del líquido, la resistencia propia del filtro y la resistencia específica de las colas a la filtración. Esta última depende del tamaño de las partículas sólidas, de la distribución granulométrica, de la forma de las partículas y de la acción de agentes floculantes (Wakeman, 2007). La resistencia al filtrado es variable a lo largo de la explotación de un yacimiento, ya que las características de las partículas sólidas dependen de las de la roca explotada como mineral, y estas a su vez pueden cambiar conforme avanza la extracción (por ejemplo, cuando varía el contenido de minera- les de arcilla). Por estos motivos, la disminución en la cantidad de agua que puede lograrse con la filtración es limitada y además variable en un rango relativamente amplio en una misma explotación (Crystal et al., 2018).

La Figura 1 presenta un modelo conceptual del proceso que siguen las colas filtradas en su disposición. Se pueden distinguir cuatro etapas en ese proceso. La etapa 1 transcurre en la planta, donde ocurre el filtrado. Las colas, que terminan el proceso de extracción con proporciones sólido líquido 1:2 a 1:6 en volumen (humedad gravimétrica entre 80 y $200 \%$ ), por el filtrado reducen su humedad a valores entre 20 y $45 \%$ (Wang et al, 2014). Esa humedad, $w_{f}$ es con la que se transportan y vierten las colas en el depósito. Allí se inicia la etapa 2, en la que las colas depositadas en la superficie del depósito experimentan una serie de procesos físicos que pueden incluir evaporación, Iluvia, congelamiento y derretimiento, infiltración, escurrimiento y operaciones de manejo de las colas, como perfilado con topadora, arado para acelerar su secado (Caldwell y Crystal, 2015) o compactación (Lara et al, 2013), etc. Como resultado de estos procesos, la humedad puede aumentar o disminuir. Su valor final, $w_{0}$ es la humedad que tienen las colas al momento de ser enterradas bajo la siguiente tongada de residuos.

Al ser cubiertas las colas con la siguiente tongada se inicia la etapa 3. Por un lado, la interacción con la atmósfera se interrumpe, ya que los fenómenos que intervienen están circunscriptos a la denominada capa activa, generalmente limitada a unos pocos decímetros de profundidad (este trabajo, Garino y Oldecop, este volumen). Por otra parte, esto es particularmente cierto para el diseño adoptado en el caso de estudio de este trabajo (ver siguiente sección), ya que el efecto de barrera capilar provoca que la humedad tienda a permanecer atrapada en la misma capa en lugar de infiltrar hacia abajo o ascender por capilaridad (Oldecop et al, 2017). Por ello se puede suponer que durante la etapa 3, la humedad ya no cambia y el proceso principal es la consolidación bajo el peso de las subsiguientes tongadas de colas. La tensión aplicada crece en forma continuada hasta el final de la construcción. Por lo tanto, la etapa 3 finaliza con la culminación de la construcción del depósito. La reducción del volumen de poros a humedad prácticamente constante determina que el grado de saturación aumenta desde $S_{0}$, el valor de enterramiento, a un valor que depende de la tapada total y del peso unitario de las colas (Lupo y Hall, 2010). La etapa 4 corresponde al cierre del depósito. Provisto de una cubierta y sistema de evacuación de escorrentía superficial adecuados, el contenido de agua global del depósito no debería cambiar, aunque pueda ocurrir redistribución espacial del agua debida a gradientes de potencial. Por tanto, en el cierre, los cambios de humedad y grado de saturación de las colas deberían ser moderados a nulos. A largo plazo, el grado de saturación tenderá a un valor $S_{c^{\prime}}$ constante en el tiempo. El objetivo del diseño es que $S_{c}$ tenga un 
valor adecuado, menor al $100 \%$ para asegurar la condición no saturada, evitando valores excesivamente bajos que faciliten la difusión del oxígeno del aire.

Considerando el proceso descripto en la Figura 1, se descubre que una variable fundamental del problema es $w_{0}$, la "humedad objetivo". Se puede definir como la humedad que permite proceder a cubrir las colas (fin de la etapa 2) con la certeza de que al finalizar la construcción se obtendrá un grado de saturación final, $S_{c^{\prime}}$ adecuado. Por lo tanto, $w_{0}$ estará determinado por la reducción de volumen de poros que experimenten las colas a lo largo de la construcción, que a su vez depende de la compresibilidad de las colas y de la tapada en cada punto del depósito. Definido un valor de $w_{0}$ bajo estas hipótesis, se puede comparar este valor con la humedad de salida de planta $w_{f}$ En el caso que resultara menor, las colas podrían ser enterradas inmediatamente. Pero como ya se explicó antes, por razones técnicas y económicas, la cantidad de secado que puede lograrse en el filtro es limitada. Por ello, es frecuente que las colas requieran de un secado adicional para llegar a $w_{0}$. En zonas áridas, con déficit hídrico, ese secado adicional puede lograrse mediante un tiempo de espera adecuado (etapa 2) para que se produzca la evaporación del exceso de humedad. Se pueden también incorporar manejos de las colas, como la remoción periódica con arado, para acelerar el secado.

Los condicionantes habituales para el diseño de un depósito de colas filtradas son la tasa de producción de la planta y el área superficial disponible para la extensión en el depósito. El espesor de tongada (capa) también está limitado a un rango adecuado para la forma de transporte y vertido escogida. Estas variables determinan el tiempo que transcurre entre el vertido de una capa y su enterramiento bajo la siguiente capa. Ese tiempo debería resultar mayor al tiempo de espera en la etapa 2, necesario para lograr la humedad $\left(w_{0}\right)$ adecuada. Debería, además, tener un margen de seguridad adicional para acomodar eventos inesperados, como aportes extraordinarios de agua debido a lluvias o problemas de funcionamiento de los filtros que producen colas con una humedad superior a la esperada. El diseño de un depósito de colas de este tipo requiere entonces contemplar todos los condicionantes enumerados: geométricos, mecánicos y de interacción con la atmósfera, para encontrar una solución técnica y económicamente viable.

La experiencia en construcción y operación de depósitos de colas filtradas es relativamente escasa. En 2010 había en el mundo aproximadamente 30 proyectos que utilizaban esta tecnología (Davies, 2011). Es frecuente en este tipo de proyectos adoptar como humedad objetivo un valor idéntico al límite de la capacidad filtrante del equipo (típicamente $15 \%$ ) que especifica el fabricante del filtro (Crystal et al., 2018). Al hacer esto no se tienen en cuenta los múltiples factores que afectan su rendimiento, ni tampoco su variabilidad. También se desprecia el cambio de humedad que ocurre en el proceso de transporte y vertido, ya sea que ésta aumente o disminuya. La consecuencia es que en muchos casos la humedad objetivo establecida en el proyecto pierde validez como parámetro de

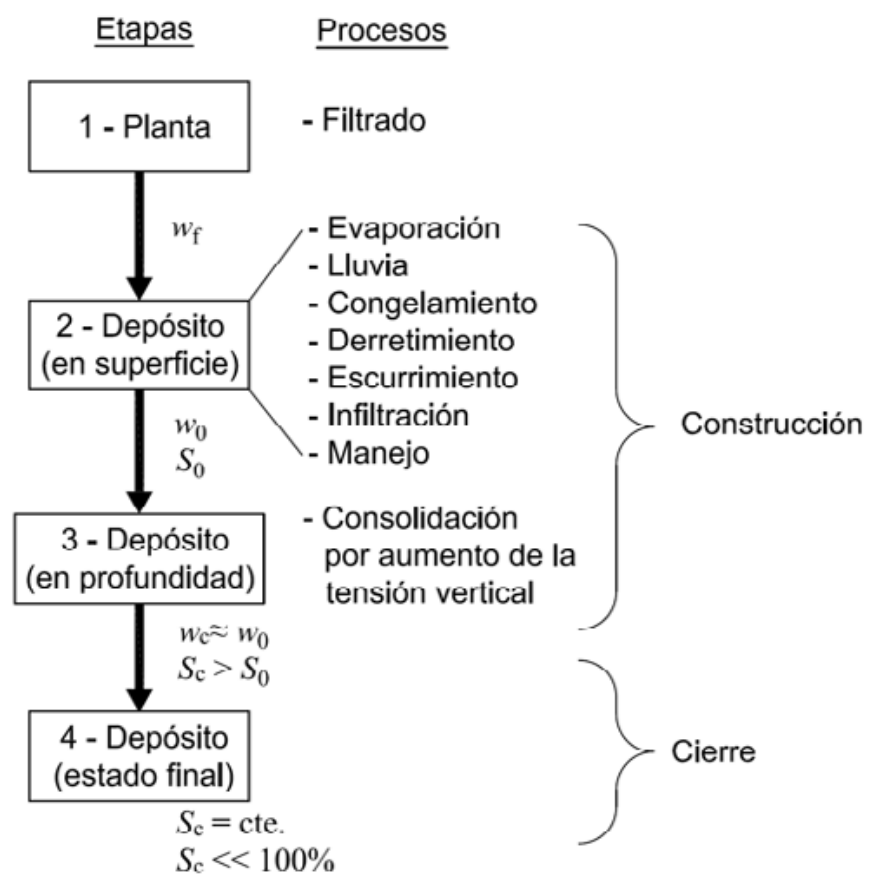

\begin{tabular}{|c|}
\hline$\underline{\text { REFERENCIAS }}$ \\
\hline$w_{\mathrm{f}}=$ humedad a la salida del filtro \\
\hline $\begin{aligned} w_{0}= & \text { humedad objetivo en el momento de } \\
& \text { cubrir las colas con la siguiente tongada }\end{aligned}$ \\
\hline $\begin{aligned} S_{0}= & \text { grado de saturación en el momento de } \\
& \text { cubrir las colas con la siguiente tongada }\end{aligned}$ \\
\hline$w_{\mathrm{c}}=$ humedad en la ctapa de cicrre \\
\hline$S_{\mathrm{c}}=$ grado de saturi \\
\hline
\end{tabular}

Figura 1. Etapas de la disposición de colas filtradas.

Figure 1. Filtered tailings disposal stages. 
operación porque, temporal o permanentemente, es imposible de alcanzar.

En este trabajo se analizan datos obtenidos en la mina Casposo (San Juan, Argentina), con el fin de evaluar la aplicabilidad del modelo conceptual antes propuesto y su utilidad para sistematizar la operación de un depósito de colas filtradas. En primer lugar, se presentan una serie de datos de campo que ilustran cómo ocurre el proceso de secado mientras las colas permanecen en la superficie del depósito (Etapa 2), para dos procedimientos diferentes de deposición: volcado sin manejos posteriores y volcado con esparcido y arado. Luego se presentan también los resultados de una serie de ensayos edométricos de las colas, realizados con el objetivo de estudiar su compresibilidad y la evolución de su grado de saturación a medida que crece la tensión vertical aplicada (etapa 3). Finalmente se discuten las posibilidades de aplicación del marco conceptual propuesto.

\section{El caso de estudio}

La mina Casposo se ubica en la vertiente argentina de los Andes centrales. Produce aleación semi-pura de oro y plata mediante molienda del mineral y lixiviación con cianuro en tanque. Una vez disueltos los metales, los residuos sólidos se concentran en tanques espesadores, donde se agregan aditivos floculantes sintéticos para mejorar la recuperación de la solución enriquecida. Los residuos espesados finalmente se filtran mediante aplicación de vacío en bandas transportadoras (Fig. 2). Las colas filtradas se transportan en camiones hasta el depósito, ubicado a aproximadamente 500 metros de la planta.

El depósito de colas consiste en una pila en donde se alternan capas de colas y capas de estéril de mina, ambas con espesor nominal de 1 metro, vertidas sin compactación. El estéril es un material tipo escollera

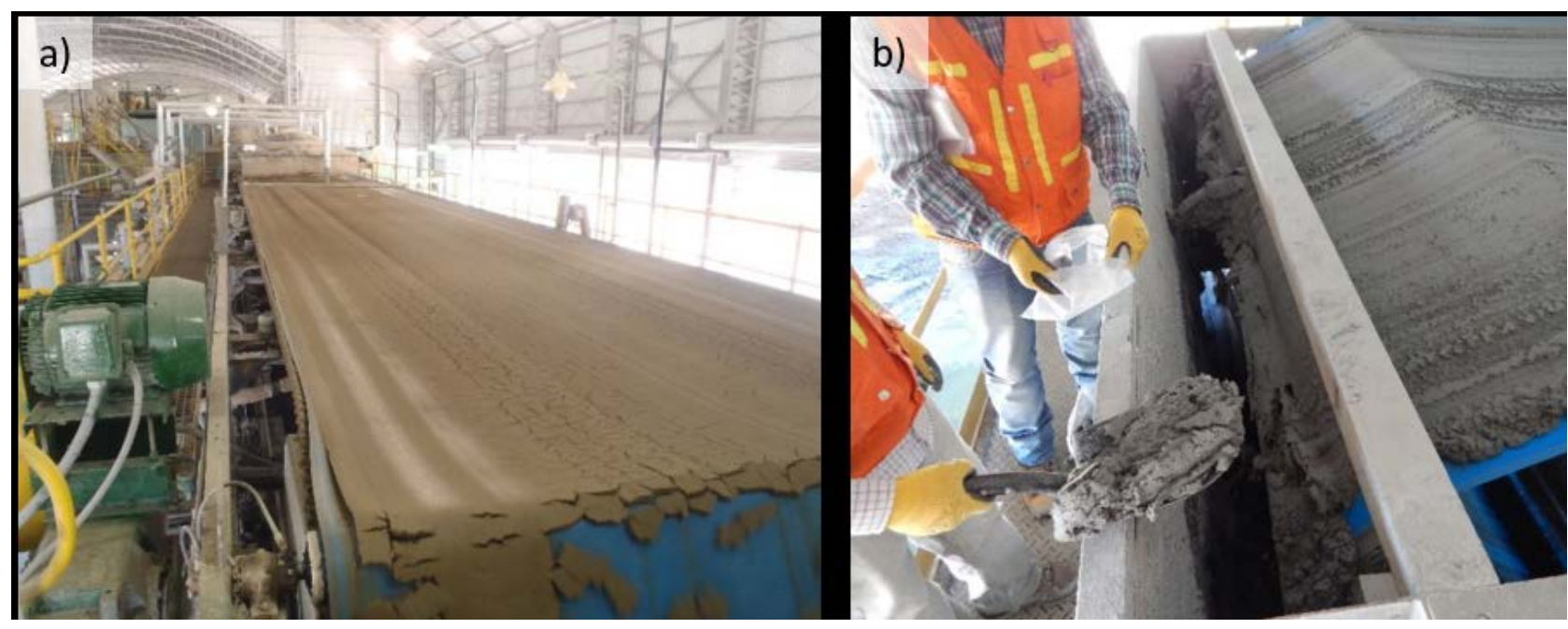

Figura 2. a) Filtro de banda y b) obtención de muestras para caracterización mecánica y seguimiento del contenido inicial de agua. Figure 2. a) Vacuum belt filter and b) tailings sampling for mechanical characterization and monitoring of the initial water content.

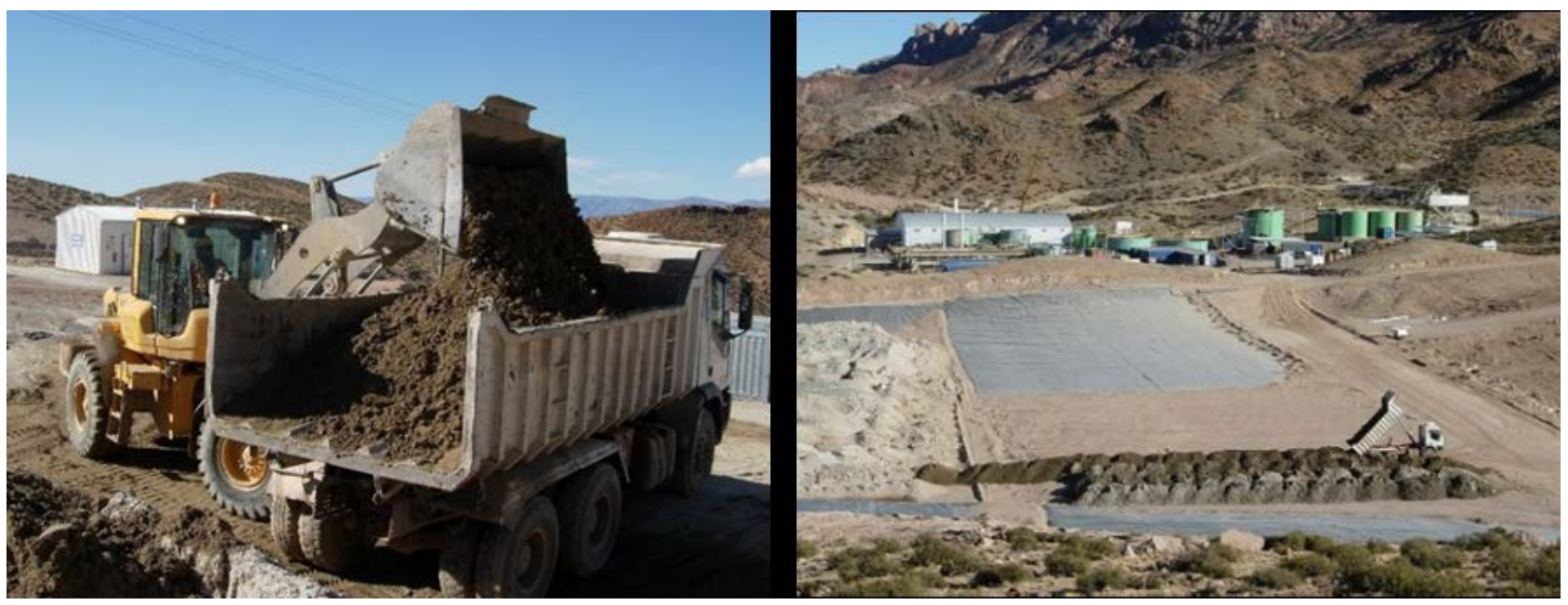

Figura 3. Transporte y volcado de las colas filtradas en el depósito. Figure 3. Hauling and dumping of filtered tailings in the deposit. 
con granulometría bastante variable y tamaños máximos de hasta $40 \mathrm{~cm}$. Los objetivos de las capas de estéril son permitir el drenaje, evitando la acumulación de presiones de poro, y mejorar la resistencia al corte promedio del material. Este diseño le confiere al depósito flexibilidad para absorber la variabilidad en la humedad de las colas y aportes de agua por lluvia o derretimiento de nieve.

La disposición de las colas se hace en la superficie de coronamiento de la pila, siguiendo un orden preestablecido, formando camellones (Figura 3). Los ciclos de trabajo están compuestos del vertido de una capa de colas, un tiempo de espera para lograr el secado necesario y luego el vertido de una capa de estéril, que además permite generar una nueva superficie transitable para los camiones que deben iniciar un nuevo ciclo de vertido (Figura 4).

El clima en el área del caso de estudio es de tipo desértico, con fuerte déficit hídrico. La precipitación media anual es de entre 100 y $200 \mathrm{~mm}$ (Bianchi and Cravero, 2010), mientras que la evapo-transpiración potencial media anual en la zona ha sido estimada en $1276 \mathrm{~mm}$ (Oldecop et al, 2010). Las mayores precipitaciones ocurren en los meses de verano (diciembre a marzo) en forma de tormentas intensas de corta duración. Las nevadas son poco frecuentes y la acumulación de nieve rara vez excede los $5 \mathrm{~cm}$. Desde 2006 se dispone de datos meteorológicos registrados en el sitio de emplazamiento del caso de estudio. En la Tabla 1 se resumen las precipitaciones medias y máximas para los distintos meses del año, abarcando el período enero de 2006 a enero de 2018. Se incluyen también datos de evaporación en tanque tipo " $\mathrm{A}$ " para el periodo 2008-2011.

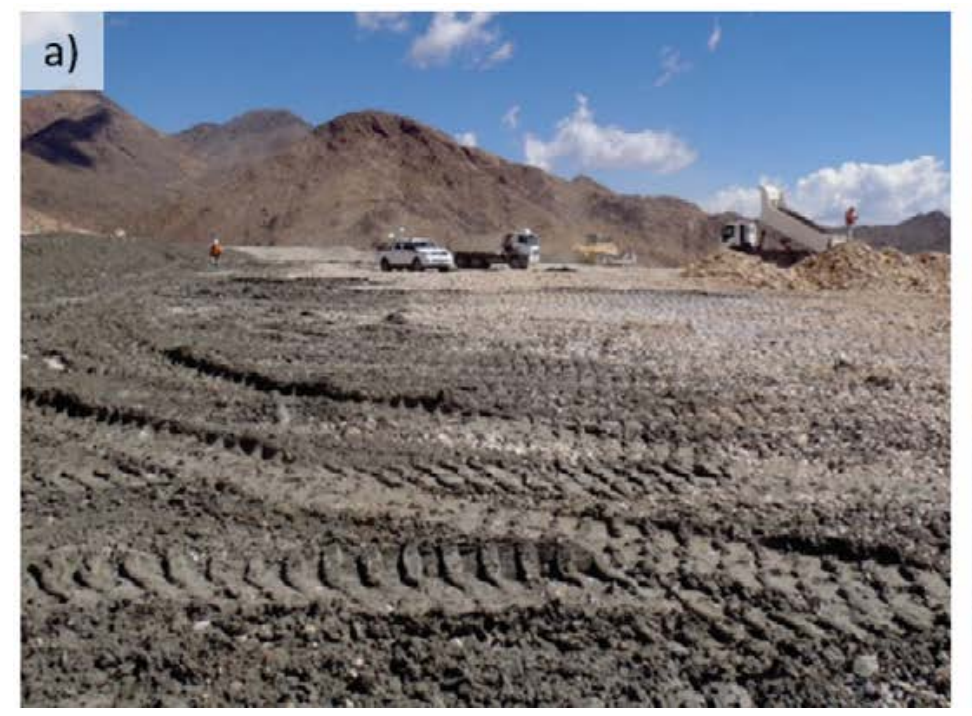

\section{Caracterización del secado de las colas expuestas a} la atmósfera

En los siguientes apartados se presentan datos de campo que describen la variación de la humedad de las colas filtradas en el tiempo, mientras están en contacto con la atmósfera (Etapa 2). Se obtuvieron datos para dos métodos alternativos de disposición: 1) volcado desde camiones conformando capas de $1 \mathrm{~m}$ de espesor, que se dejan secar sin manejos adicionales y 2) volcado desde camiones y esparcido con topadora conformando capas de $40 \mathrm{~cm}$ de espesor, que se remueven diariamente por arado para incrementar la capacidad de secado por interacción con la atmósfera.

\section{Colas volcadas desde camión, sin manejos adicionales}

Se tomaron muestras para determinación de humedad mediante secado en estufa (ASTM D2216-19) de las colas, con diferente tiempo de permanencia en la superficie del depósito. Se adoptó la convención de tomar muestras en superficie y a $50 \mathrm{~cm}$ de profundidad (a media altura de la capa). En la Figura 5a se presentan los resultados de estas mediciones realizadas a lo largo de los años 2011 a 2013. No se discriminan los datos por estación del año, sólo por profundidad de muestreo. Se grafican el parámetro "humedad gravimétrica" ( $w$, water content), habitual en mecánica de suelos y también, en eje secundario, la fracción másica de agua ( $w^{\prime}$ water mass fraction), definido como el peso de agúa contenida en las colas dividido en el peso de la mezcla de partículas sólidas y agua. En las plantas de procesamiento de mineral es mucho más habitual e intuitivo el uso del segundo parámetro que del primero. De todas formas, ambos parámetros

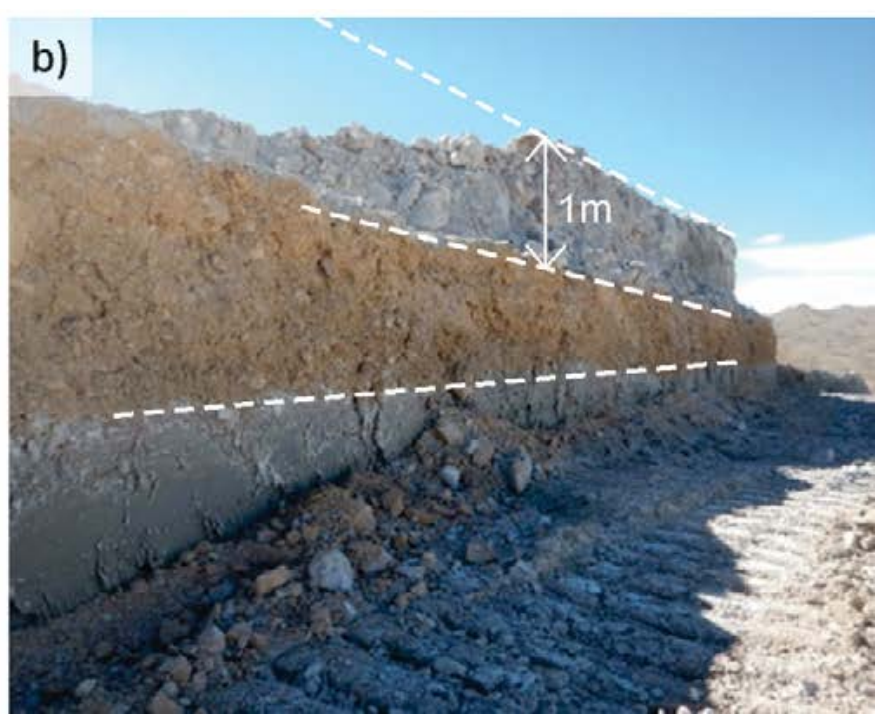

Figura 4. a) Vertido de una nueva capa de colas sobre capa de estéril y b) alternancia de capas de colas y de estéril de aproximadamente 1 $m$ de espesor expuesta en una excavación temporal.

Figure 4. a) Placing a new tailings layer over a waste rock layer and b) alternated nearly $1 \mathrm{~m}$ thick layers of tailings and waste rock exposed in a temporary excavation (right). 
Rodari, G., et al, 2021. Secado de colas filtradas en contacto con la atmósfera, 132 (4): 551-571

\begin{tabular}{c|ccccccccccccc}
\hline Emm] & Ene & Feb & Mar & Abr & May & Jun & Jul & Ago & Sep & Oct & Nov & Dic & Anual \\
\hline $\begin{array}{c}\text { Precip. Media } \\
\text { Periodo: 2006-18 }\end{array}$ & 36.4 & 22.7 & 11.6 & 2.0 & 7.0 & 6.6 & 8.8 & 9.4 & 4.9 & 2.9 & 13.6 & 13.0 & 138.9 \\
& & & & & & & & & & & & & \\
$\begin{array}{c}\text { Precip. Máxima } \\
\text { Periodo: 2006-18 }\end{array}$ & 154 & 64.4 & 50.8 & 10.6 & 26.4 & 22.4 & 61.2 & 39.0 & 14.1 & 8.8 & 45.9 & 42.4 & 265.5 \\
$\begin{array}{c}\text { Evap. tanque tipo A } \\
\text { Periodo: 2008-11 }\end{array}$ & 280 & 219 & 192 & 180 & 138 & 124 & 140 & 168 & 188 & 250 & 330 & 304 & 2513
\end{tabular}

Tabla 1. Precipitaciones medias, precipitación máxima y evaporación media medida en tanque tipo " $A$ ", en el sitio de la Mina Casposo. Table 1. Mean rainfall, maximum rainfall and mean evaporation measured in class " $A$ " pan at Casposo mining site.

son equivalentes y además la conversión se hace fácilmente con las expresiones:

$$
w=P_{w} / P_{s o l} ; w^{\prime}=P_{w} /\left(P_{s o l}+P_{w}\right)
$$

Con peso de agua (weight of water) y peso de las partículas sólidas (weigth of solid particles) y

$$
w=w^{\prime} /\left(1-w^{\prime}\right) ; w^{\prime}=w /(1+w)(2)
$$

La primera característica notable del conjunto de datos obtenidos es su variabilidad. Los factores que pueden determinar o influir en esa variabilidad son múltiples: composición mineral y granulométrica de las colas, parámetros de operación de la planta y del filtro, agentes meteorológicos y sus variaciones diarias y estacionales y procedimiento de vertido. Entre las series de datos, de muestras de la superficie y a 50 $\mathrm{cm}$ de profundidad, es notable la diferencia en la tasa de secado. Además, los datos sugieren que las muestras a $50 \mathrm{~cm}$ alcanzan un punto de interrupción del secado, cosa que no se observa en las muestras superficiales. En algunas muestras obtenidas en capas de colas que permanecieron en Etapa 2 durante largos periodos (hasta 180 días) debido a circunstancias particulares de operación del depósito, se observa que efectivamente la tendencia es a una humedad constante, o más bien a un rango de humedad constante.
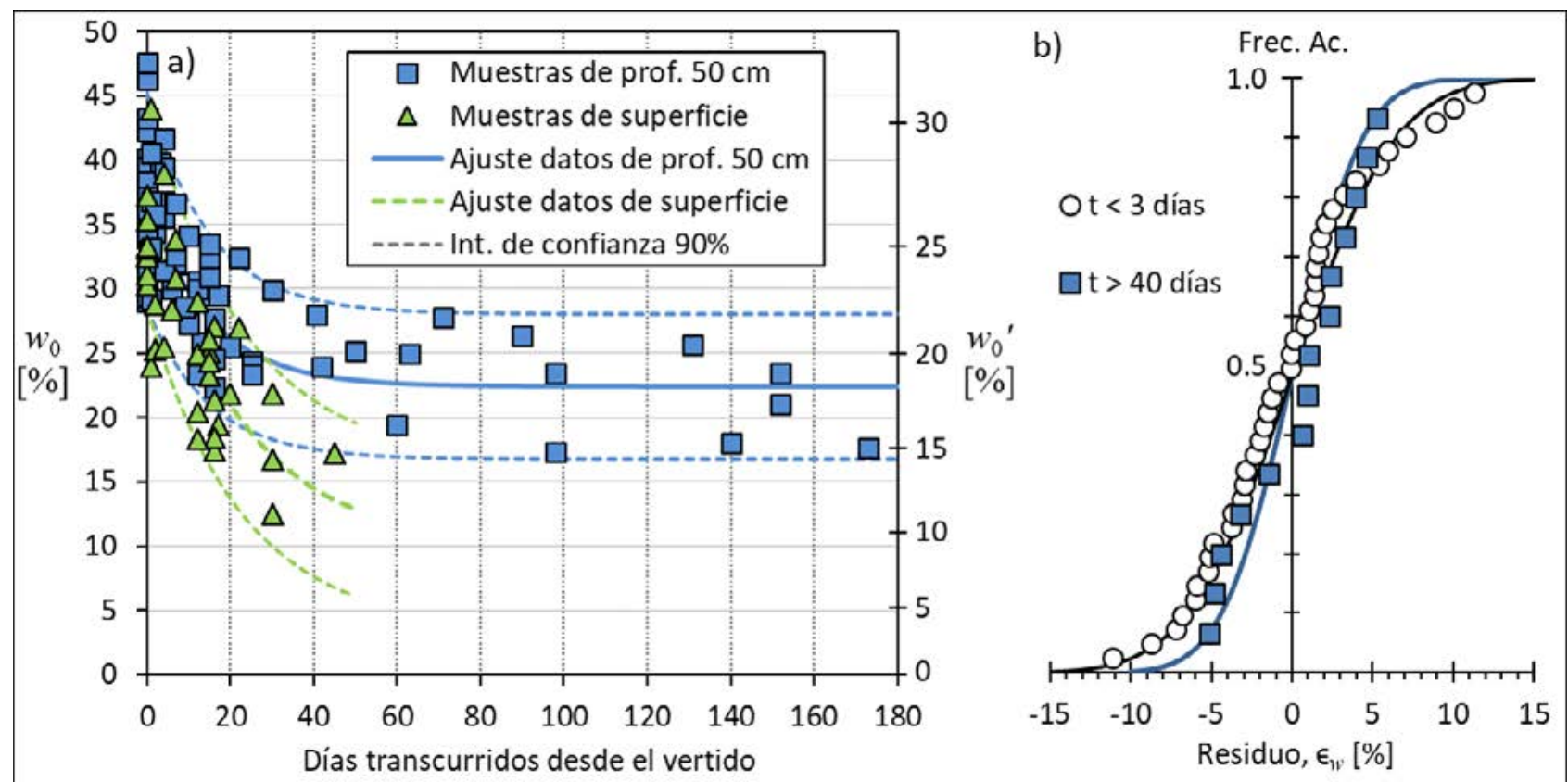

Figura 5. a) Humedad medida sobre muestras obtenidas en capas de colas sin manejo posterior al vertido en etapa 2, a diferentes tiempos transcurridos desde el vertido, en superficie y a $50 \mathrm{~cm}$ de profundidad. b) Análisis de varianza del residuo del ajuste de la Ecuación (3) en dos segmentos del proceso de secado: 1) dos primeros días y 2) después de 40 días.

Figure 5. a) Water content measured in samples taken from the surface and at $50 \mathrm{~cm}$ depth of tailings layers at stage 2, with no handling after placement and at different times since placement. b) Variance analysis of the residual from the adjustment of equation (3) for two time-spans of the drying process: 1) first two days and 2) after 40 days. 
El motivo más probable de la interrupción en el secado en profundidad de las colas sin manejo posterior al vertido, es la interrupción del ascenso capilar por desarrollo de una capa superficial muy seca, de unos pocos decímetros de profundidad (Garino y Oldecop, este volumen; Garino, 2018). Este mecanismo también se puede deducir de la observación de una serie de perfiles de humedad determinados en calicatas excavadas en capas de colas en etapa 2. Estos datos se muestran en la Figura 6, junto a valores de peso unitario determinados in situ mediante el método del cono de arena (ASTM D1556). Los datos de peso unitario no muestran una tendencia definida ni una dependencia clara con el tiempo transcurrido desde el vertido. Los perfiles de humedad para diferentes tiempos transcurridos desde el vertido, sugieren que la capa de colas parte de una distribución inicial de humedad prácticamente uniforme en su espesor. El secado se desarrolla desde la superficie y también, en menor medida y durante el período de tiempo inicial, desde el contacto con la capa subyacente de estéril, tal como se puede apreciar en el perfil correspondiente a 15 días de la Figura 6. Esto se debe a que el estéril se encuentra inicialmente seco y por lo tanto absorbe algo de humedad, Aproximadamente a los 50 días, el perfil de humedad alcanza un límite inferior. Los perfiles obtenidos para tiempos de secado mayores (150 y 290 días) no muestran disminuciones adicionales de la humedad en profundidad. De hecho, en superficie muestran incrementos de hu- medad respecto del perfil de 50 días. Lo más probable es que esto se deba a un rehumedecimiento por Iluvias ocurridas antes de relevados esos perfiles. Esta observación pone en evidencia la dificultad de comparar medidas puntuales de humedad tomadas en distintos puntos del depósito y diferentes momentos, dada la variedad de factores que afectan a las medidas. Sin embargo, dejando de lado los efectos de las lluvias (aparentemente superficiales), la existencia de un perfil límite inferior de humedad de la capa es consistente con la evolución a largo plazo que muestra la Figura 5a para las muestras de $50 \mathrm{~cm}$ de profundidad. Se puede observar en la Figura 6 que el perfil de humedad a 50 días se aproxima bien mediante una distribución parabólica y que el valor a $50 \mathrm{~cm}$ de profundidad es cercano o algo mayor al valor medio de la capa. Por lo tanto, la medida de la humedad a $50 \mathrm{~cm}$ de profundidad es, en este caso, un buen indicador de la humedad promedio en la altura de la capa.

Se propone la siguiente expresión matemática para ajustar la evolución temporal de la humedad:

$$
w_{0}=(a-b) e^{-c t}+b+\epsilon_{w}(0, s)
$$

donde $\mathrm{w}_{0}$ es una variable aleatoria que describe la variación de la humedad en el tiempo a una cierta profundidad de referencia; $t$ es el tiempo transcurrido
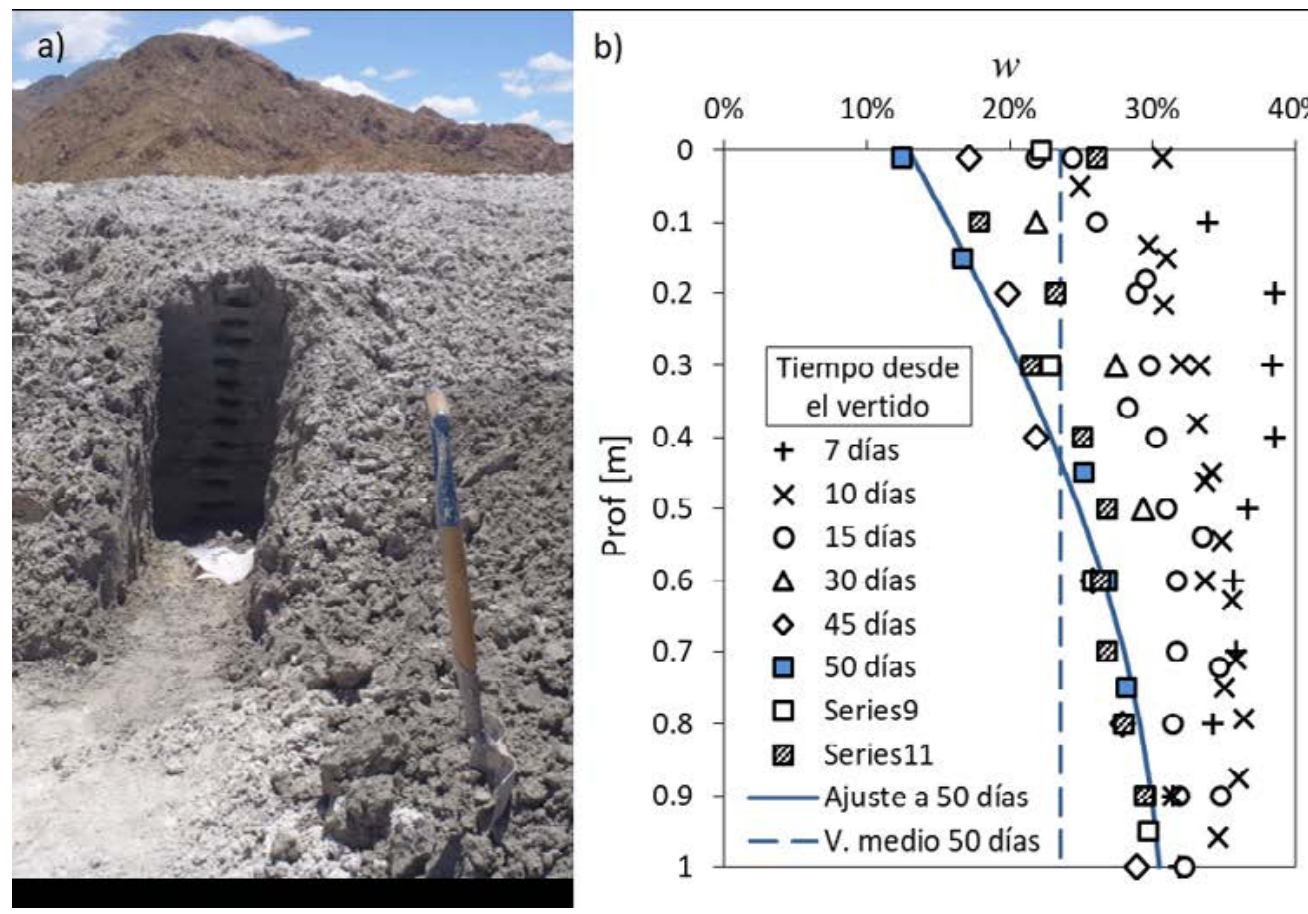

c) $\gamma_{a s}\left[\mathrm{kN} / \mathrm{m}^{3}\right]$

$\begin{array}{llllll}0 \% & 10 & 11 & 12 & 13 & 14\end{array}$

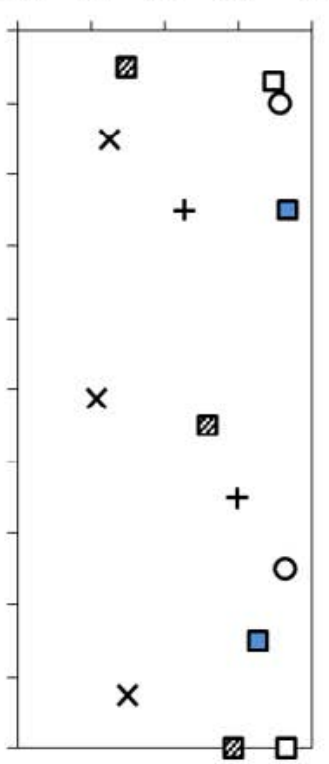

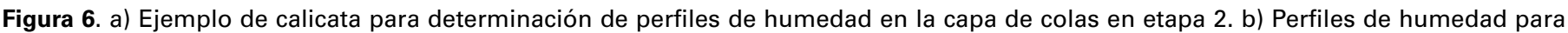

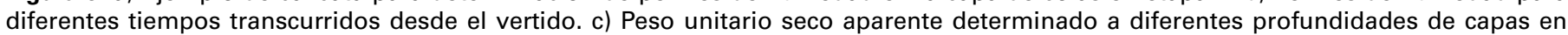
etapa 2 y para diferentes tiempos transcurridos desde el vertido.

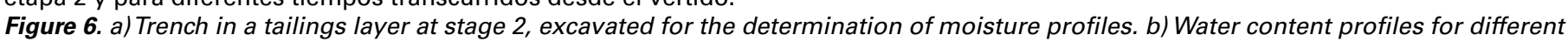

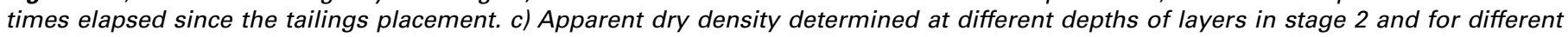
times elapsed since the tailings placement. 
desde el vertido (time elapsed since placing) en días; $a, b$ y $c$ son parámetros de ajuste (fitting parameters). Los dos primeros sumandos de la Ecuación (3) dan el valor esperado (media poblacional o esperanza matemática) de la humedad, , a la profundidad de referencia dentro de la capa estudiada (a la que se hayan obtenido los datos utilizados en el ajuste), para un cierto tiempo transcurrido desde el vertido. $\epsilon_{w}$ es el residuo, es decir la diferencia, que se asume una variable aleatoria normalmente distribuida con media nula y desviación estándar.

Los parámetros de la Ecuación (3) se obtuvieron para cada grupo de datos mostrado en la Figura 5, es decir de muestras de superficie y de muestras tomadas a 50 $\mathrm{cm}$ de profundidad, mediante regresión por mínimos cuadrados. Los parámetros obtenidos del ajuste se resumen en la Tabla 2. El valor (a-b).c indicado en la última columna de la Tabla 2 es la tasa de secado inicial (pendiente inicial, negativa, de la curva de humedad esperada). En la Figura 5a se presentan también las curvas de valor esperado para cada grupo de datos, en trazo continuo. En la Figura $5 \mathrm{~b}$ se presenta el análisis de varianza del residuo. Repitiendo este análisis para diferentes segmentos temporales, se comprueba que la desviación estándar disminuye con el paso del tiempo, por lo que se determinó un valor inicial, $s_{i}$ y uno de largo plazo, $s_{u}$. Para graficar los intervalos de confianza de $90 \%$ mostrados en la Figura 5 a, se asumió una variación de la desviación estándar dada por una ecuación similar a la Ecuación (3):

$$
s=\left(s_{i}-s_{u}\right) e^{-c t}+s_{u}(4)
$$

Donde el valor del parámetro $c$ es el mismo que en la Ecuación (3). Los valores de $s_{i}$ y $s_{u}$ también se indican en la Tabla 2. Los parámetros de la Tabla 2 junto con las Ecuaciones (3) y (4) describen estadísticamente el proceso de secado de las colas vertidas sin manejo posterior.

El comportamiento observado en las Figuras 5 y 6 sugiere que una manera de mejorar el secado por evaporación es extender las colas en capas de menor espesor y removerlas para evitar la interrupción de la evaporación por la formación de la capa seca o costras debidas a la precipitación de sales.

\section{Colas volcadas, extendidas con topadora y aradas diariamente}

Se realizó una prueba piloto de un método alternativo de vertido. Las colas correspondientes a cada día de producción se transportaron con camión y se volcaron en sectores previamente asignados y delimitados, denominados celdas. Luego se extendieron con topadora y se removieron una vez al día mediante un arado de discos, comenzando el proceso el día posterior al vertido (Figura 7). El espesor de las capas debió limitarse a la profundidad de volteo del arado de discos empleado $(40 \mathrm{~cm})$. Esto implica un mayor uso de superficie de trabajo en comparación con el método que se describió en el apartado anterior. El objetivo de este manejo es acelerar el secado para conseguir reducir tiempo de permanencia del depósito en la Etapa 2.

La humedad de las colas se midió diariamente en muestras extraídas a $20 \mathrm{~cm}$ bajo la superficie (media altura de la capa). Los datos obtenidos se muestran

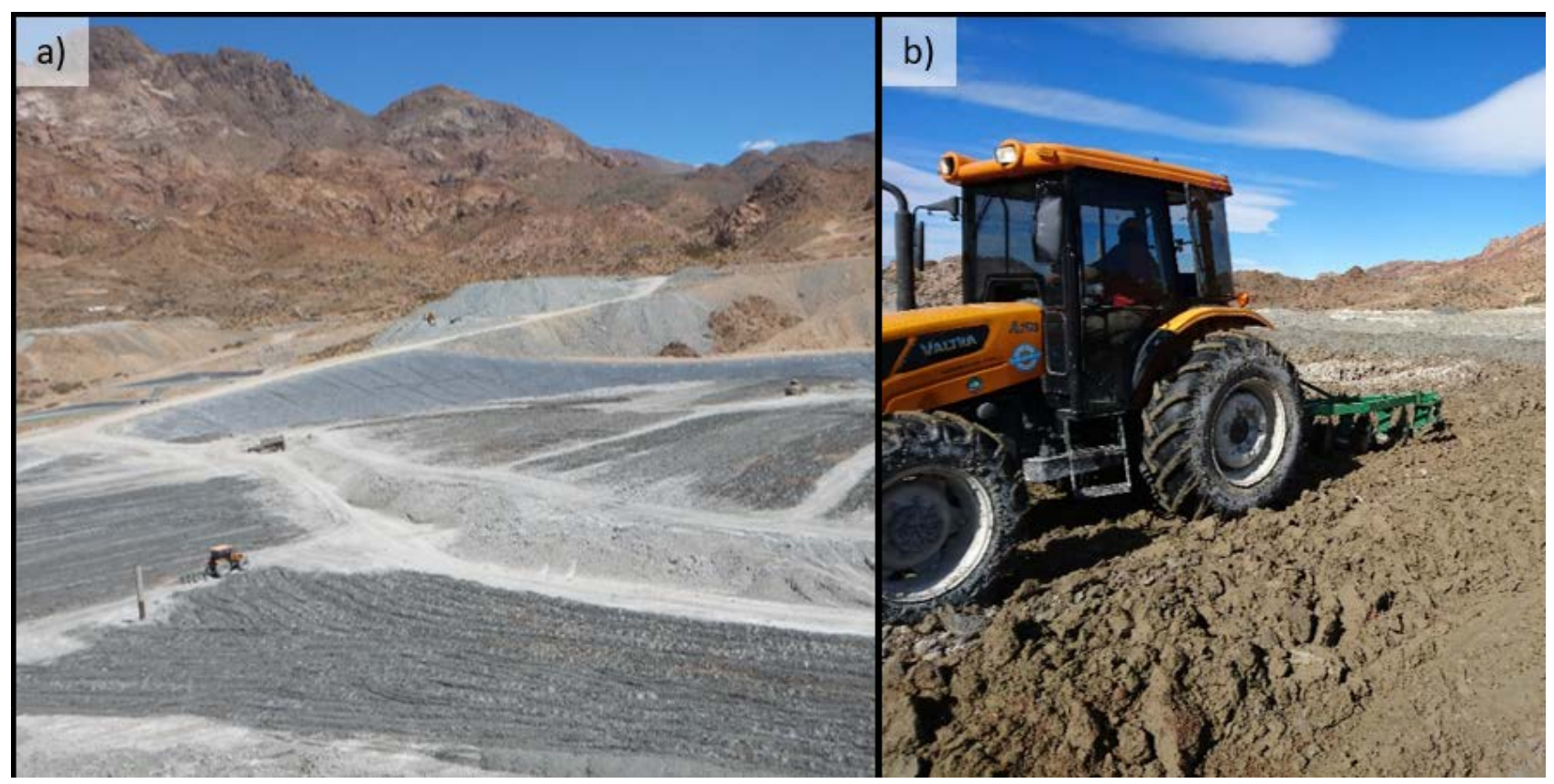

Figura 7. Deposición de colas por volcado, extensión y arado: a) celdas y b) maquinaria de arado.

Figure 7. Tailings placement by dumping, spreading and ploughing: a) cells and b) ploughing equipment. 

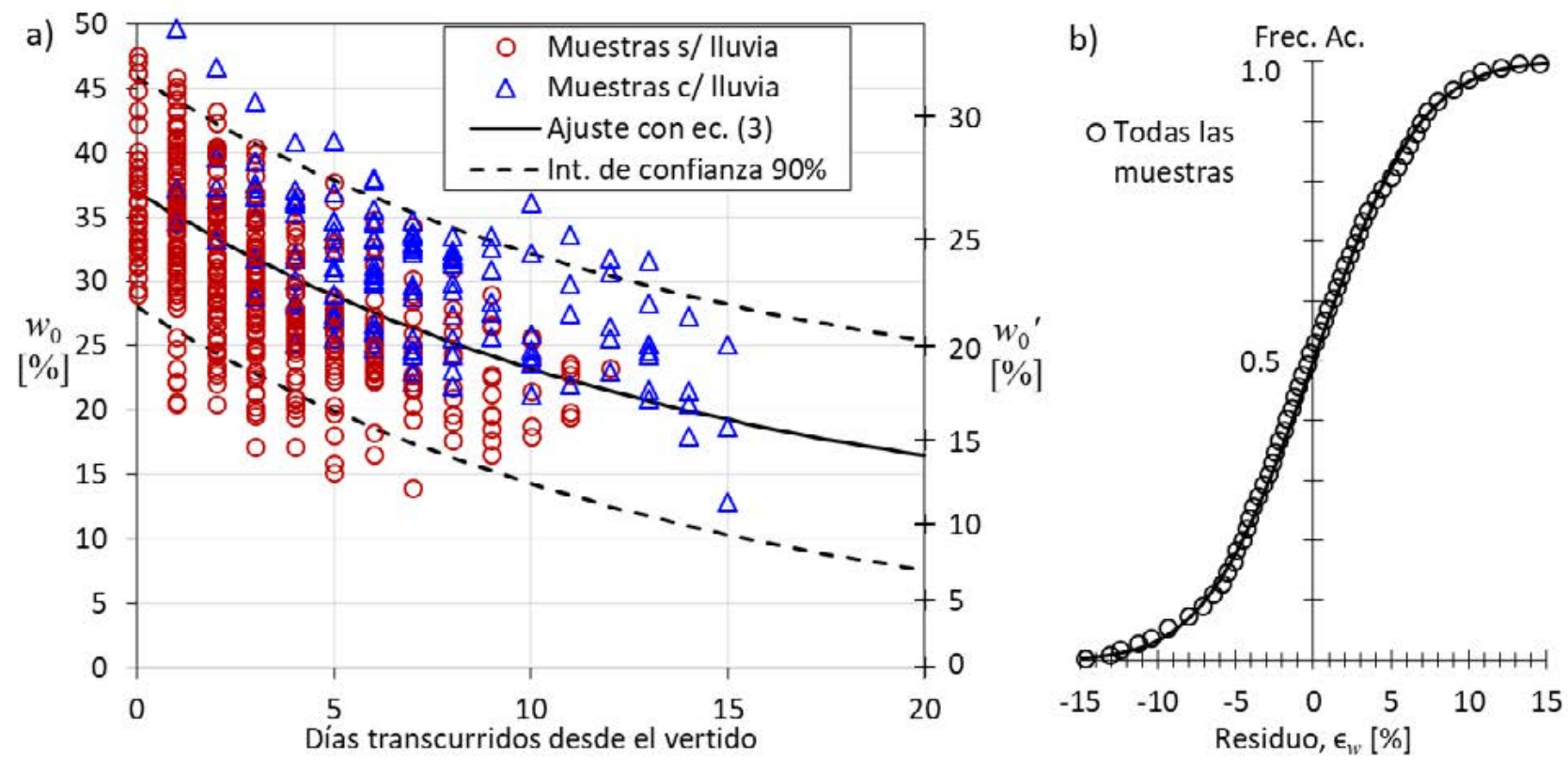

Figura 8. a) Humedad en función del tiempo transcurrido desde el vertido para colas volcadas, extendidas con topadora en capas de 0.4 $\mathrm{m}$ de espesor y aradas diariamente. Ajuste con la Ecuación (3) (parámetros indicados en la Tabla 2). b) Análisis de varianza del residuo del ajuste de la Ecuación (3).

Figure 8. a) Water content as a function of the time elapsed since placement for tailings that were spread with bulldozers in $0.4 \mathrm{~m}$ thick layers and ploughed daily. Adjustment with equation (3) (parameters are indicated in Table 2). b) Variance analysis of the residual from adjustment of equation (3).

en la Figura 8a. Se hicieron varios análisis preliminares, separando los datos por estación del año, en la hipótesis que las tasas de secado variarían significativamente entre estaciones. Si bien esto efectivamente ocurre, para el caso de estudio las típicas lluvias de verano terminan por compensar el efecto de la mayor intensidad de evaporación (ver Tabla 1). En la Figura 8a se aprecia cómo las muestras tomadas luego de una Iluvia se ubican preferencialmente en la banda superior de la serie. El resultado es que, al menos en el periodo estudiado, las tasas de secado no fueron muy diferentes en las diferentes estaciones (invierno, primavera y verano). En la Figura 8.b se presenta el análisis de varianza del residuo del ajuste de la Ecuación (3) al conjunto de datos completo. La simetría y buena aproximación a la distribución normal sugiere que, para el caso estudiado, es admisible tratar los datos como una serie única homogénea, sin tener en cuenta la estación ni las lluvias.

En la Figura 8a se presenta también la curva de valor esperado de la humedad a $20 \mathrm{~cm}$ de profundidad de la capa arada, obtenida con el ajuste de la Ecuación (3) por regresión con mínimos cuadrados. Los parámetros obtenidos en este caso se indican también en la Tabla 2. También se trazaron los límites del intervalo de confianza del $90 \%$, a partir del valor de desviación estándar determinado con el análisis de varianza del residuo que se muestra en la Figura 8.b. Se observa que el secado ocurre a tasas significativamente mayores que en el caso de las colas sin arado (tasa media inicial $90 \%$ mayor según se puede ver en la última columna de la Tabla 2). En este caso la desviación estándar no cambia significativamente con el tiempo, por lo que se adoptó un valor constante. Por otra parte, llama la atención que la variabilidad de los datos correspondientes a las colas aradas es algo mayor a la de los datos de las colas sin arado. Si bien la operación de arado tiende a uniformar la humedad en el espesor de la capa, es posible que la menor duración de los periodos de secado y el menor espesor de capa, determinen que factores externos, como las variables meteorológicas, tengan una mayor influencia en el proceso y por lo tanto determinen una mayor variabilidad en las muestras.

La Figura 9 muestra una comparación de los procesos de secado resultantes de los dos métodos de vertido aplicados, en base a las curvas de valor medio de fracción másica de agua que resultaron del ajuste de la Ecuación (3) y los límites del intervalo de confianza del $90 \%$ de cada caso. El instante en que se interrumpe el proceso de secado por vertido de una nueva capa (final de la Etapa 2) determina la humedad w0. Por ejemplo, para un periodo de 45 días de secado en Etapa 2 de las colas sin arado, se alcanza un valor medio de $=23 \%(=18.7 \%)$. El mismo valor medio se alcanza en las colas aradas al cabo de 10 días. Sin embargo, a causa de la mayor variabilidad w0 en las colas aradas, si en lugar de comparar el valor medio se compara, por ejemplo, el valor de w0 con $95 \%$ de probabilidad de no ser excedido, se requieren 14 días en el método de colas aradas para lograr lo que en las colas sin arar se logra en 45 días. 


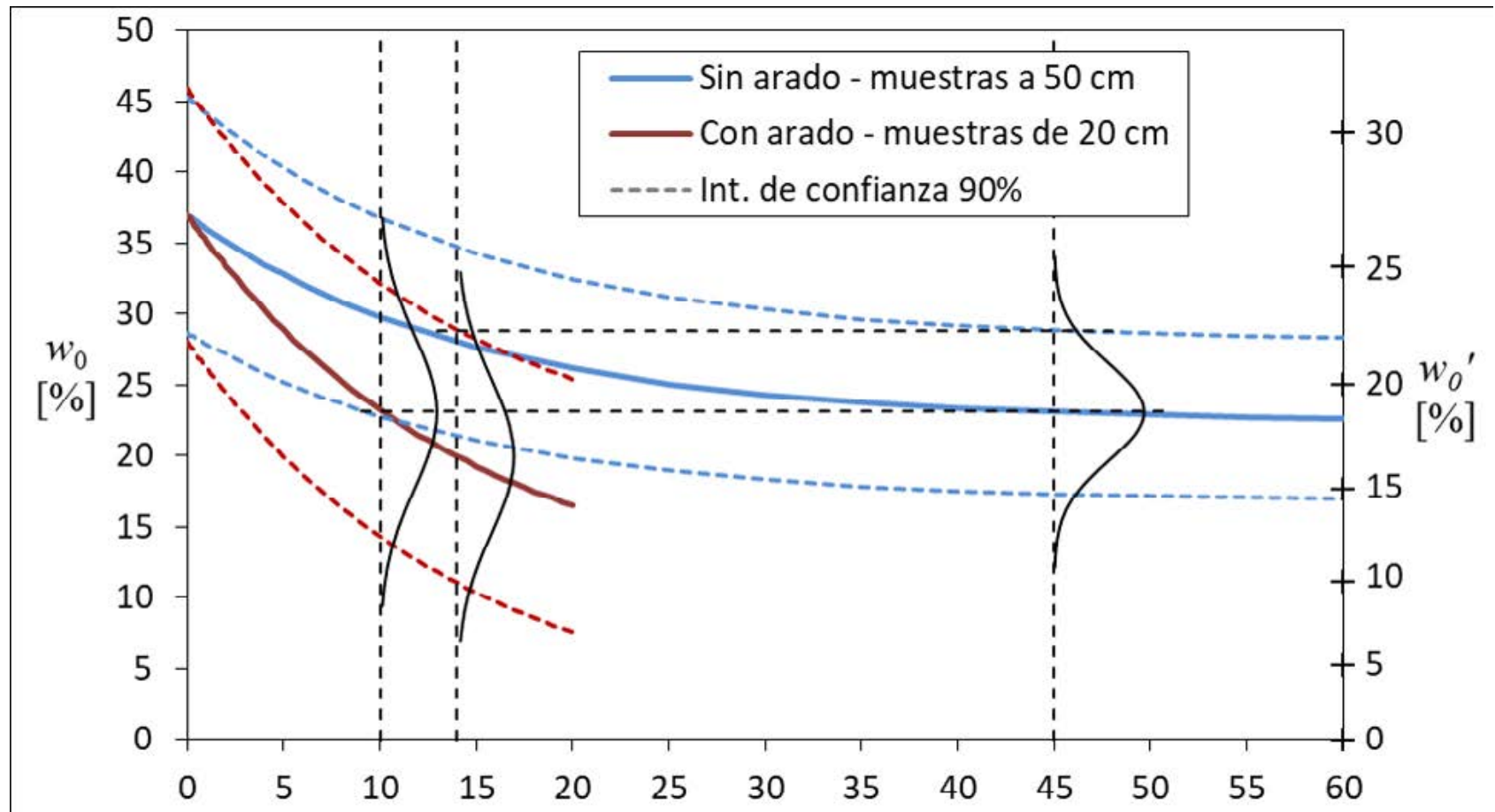

\section{Días transcurridos desde el vertido}

Figura 9. Comparación del secado en Etapa 2 para los dos métodos de vertido aplicados, en base al ajuste de la Ecuación (3) a los datos obtenidos en cada caso y los límites del intervalo de confianza del $90 \%$. Se muestran también las campanas de distribución normal para valores particulares de tiempos transcurridos, 45 días en las colas sin arado, 10 y 14 días en las colas aradas.

Figure 9. Comparison of water content at the end of stage 2 for the two placing methods, based on the adjustment of Equation (3) to experimental data and the 90\% confidence interval. The normal distribution bells are also shown for particular values of elapsed time, including 45 days for tailings without ploughing, 10 and 14 days for tailings with ploughing.

\begin{tabular}{ccccccccccc} 
Manejo & & Espesor & Profundidad & \multicolumn{5}{c}{ Parámetros de ajuste ecuaciones (3) y (4) } \\
\cline { 6 - 11 } $\begin{array}{c}\text { posterior al } \\
\text { vertido }\end{array}$ & Periodo & $\begin{array}{c}\text { Ee capas } \\
{[\mathrm{m}]}\end{array}$ & $\begin{array}{c}\text { de muestreo } \\
{[\mathrm{m}]}\end{array}$ & $a$ & $b$ & $\begin{array}{c}c \\
{[1 / \mathrm{dí}]}\end{array}$ & $s_{i}$ & $s_{u}$ & $\begin{array}{c}(a-b) . c \\
{[1 / \text { dia }]}\end{array}$ \\
\hline \multirow{2}{*}{ Ninguno } & $2011-2013$ & 1 & 0.00 & 0.369 & 0.100 & 0.045 & 0.050 & 0.040 & 0.0121 \\
& & 1 & 0.50 & 0.369 & 0.224 & 0.068 & 0.050 & 0.034 & 0.0099 \\
\hline Arado diario & $2016-2017$ & 0.4 & 0.20 & 0.369 & 0.100 & 0.071 & 0.054 & 0.054 & 0.0191 \\
\hline
\end{tabular}

Tabla 2. Secado en Etapa 2 y parámetros de ajuste de las Ecuaciones (3) y (4).

Table 2. Tailings drying at stage 2 and adjustment parameters of equations (3) and (4).

\section{Compresibilidad de las colas}

Para estudiar la compresibilidad de las colas y poder así analizar cómo evoluciona su grado de saturación por aumento de la tapada durante la construcción, se realizaron una serie de ensayos edométricos. Los materiales ensayados se identifican en la Tabla 3 , donde también se resumen algunas propiedades. El material t1 se recolectó del depósito, es decir, son colas producidas por planta en pleno funcionamiento. El material t2 es mineral molido producido en una planta piloto en la etapa de proyecto de la mina. En la Figura 10 se presentan las curvas granulométricas de los materiales $\mathrm{t} 1$ y $\mathrm{t} 2$. El edómetro utilizado tiene un añillo de $200 \mathrm{~mm}$ de diámetro interior y la altura inicial de las probetas fue de $50 \mathrm{~mm}$.

Los ensayos edométricos se realizaron sobre probetas preparadas a diferentes humedades iniciales, secando primero la muestra al aire, para luego agregarle agua necesaria para lograr la humedad inicial deseada. Por otro lado, el índice de poros inicial de cada probeta fue el resultante de colocar el material 


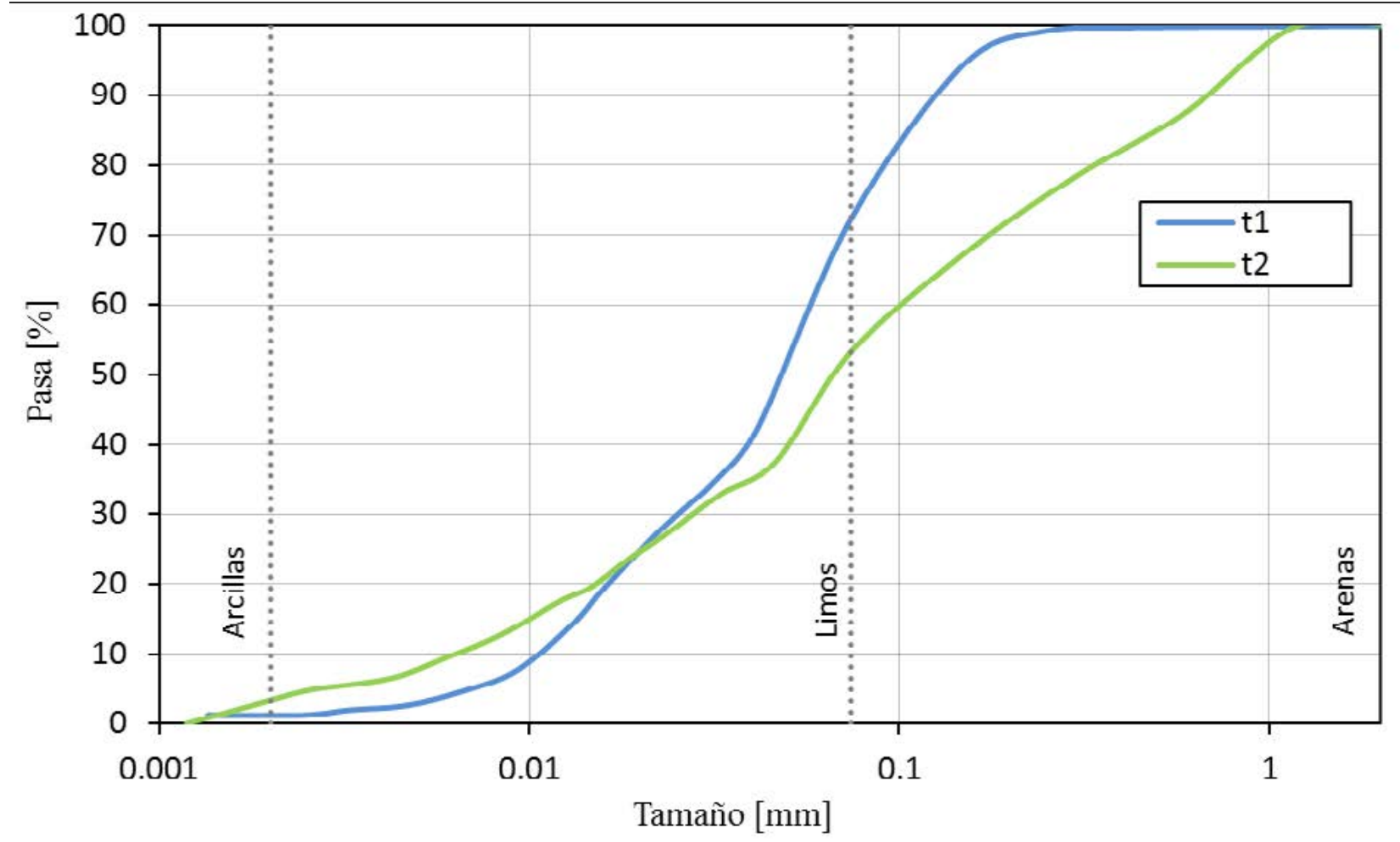

Figura 10. Granulometría de los materiales ensayados en edómetro. Figure 10. Grain size distribution of the materials tested in the oedometer.

\begin{tabular}{ccccc}
\hline Material & $\begin{array}{c}\text { Peso esp. de sólidos } \\
{\left[\mathrm{kN} / \mathrm{m}^{3}\right]}\end{array}$ & LL & LP & IP \\
\hline $\mathrm{t} 1$ & 26.12 & 20 & 18 & 2 \\
$\mathrm{t} 2$ & 26.29 & 22 & 22 & $\mathrm{NP}$
\end{tabular}

Tabla 3. Propiedades de los materiales ensayados en edómetro. Table 3. Properties of the materials tested in the oedometer.

en el equipo de ensayo sin compactación, en el estado más suelto posible. Este método de elaboración de las probetas intenta recrear las condiciones con las que las colas se colocan en el depósito.

Una primera observación interesante se puede hacer a partir de los datos de preparación de las probetas (Tabla 4). A humedades de preparación decrecientes, los índices de poro iniciales del material t2 resultaron crecientes, en tanto que en material t1 resultaron decrecientes. En el caso del material t2, este efecto puede atribuirse al esponjamiento debido a la acción de las fuerzas capilares que se desarrollan en los meniscos de agua entre las partículas sólidas. La influencia de las fuerzas capilares disminuye al aumentar la humedad, hasta anularse al alcanzar la saturación y por ello el efecto de esponjamiento disminuye con el aumento de humedad inicial. En contraste, en los especímenes elaborados con material t1, la acción del aditivo floculante provoca la agregación de los sólidos en grumos de aproximadamente $1 \mathrm{~cm}$ de diáme- tro (Figura 11a). La estructura de los grumos puede observarse aún después de finalizado el ensayo ( $\mathrm{Fi}$ gura $11 \mathrm{~b}$ ). Estos agregados, que también se observan en las colas recién salidas de la planta (Figura 11c), tienen gran capacidad de almacenar agua. Siendo así, es posible que una disminución de humedad provoque una contracción volumétrica de los grumos, haciendo que la concentración de solidos aumente y, por lo tanto, que el índice de poros disminuya.

La carga se aplicó en incrementos, manteniendo la tensión constante durante $24 \mathrm{hs}$ y registrando en forma continua la deformación vertical. Durante todo el ensayo se permitió el drenaje del agua de los poros a través de los dos cabezales del edómetro. En todos los casos, la espera de un día fue suficiente para que el proceso de deformación se detuviera o alcanzara una tasa de deformación despreciable. También, cuando ocurrió expulsión de agua, se verificó que esta se hubiera detenido antes de aplicar el siguiente incremento de carga. Las condiciones iniciales y finales de los ensayos realizados se resumen en laTabla 4.

Con la deformación de la probeta medida al final del periodo de 24 hs, se calculó la evolución del índice de poros y se trazaron las curvas de compresión virgen que se muestran en la Figura 12a. Los especímenes 1 y 2 tenían una consistencia fluida al momento de colocarlos en el edómetro. A humedades elevadas, la mezcla tiende a segregarse rápidamente. Luego del montaje se esperó $24 \mathrm{hs}$ antes de aplicar el primer incremento de carga. Durante ese periodo, estas pro- 


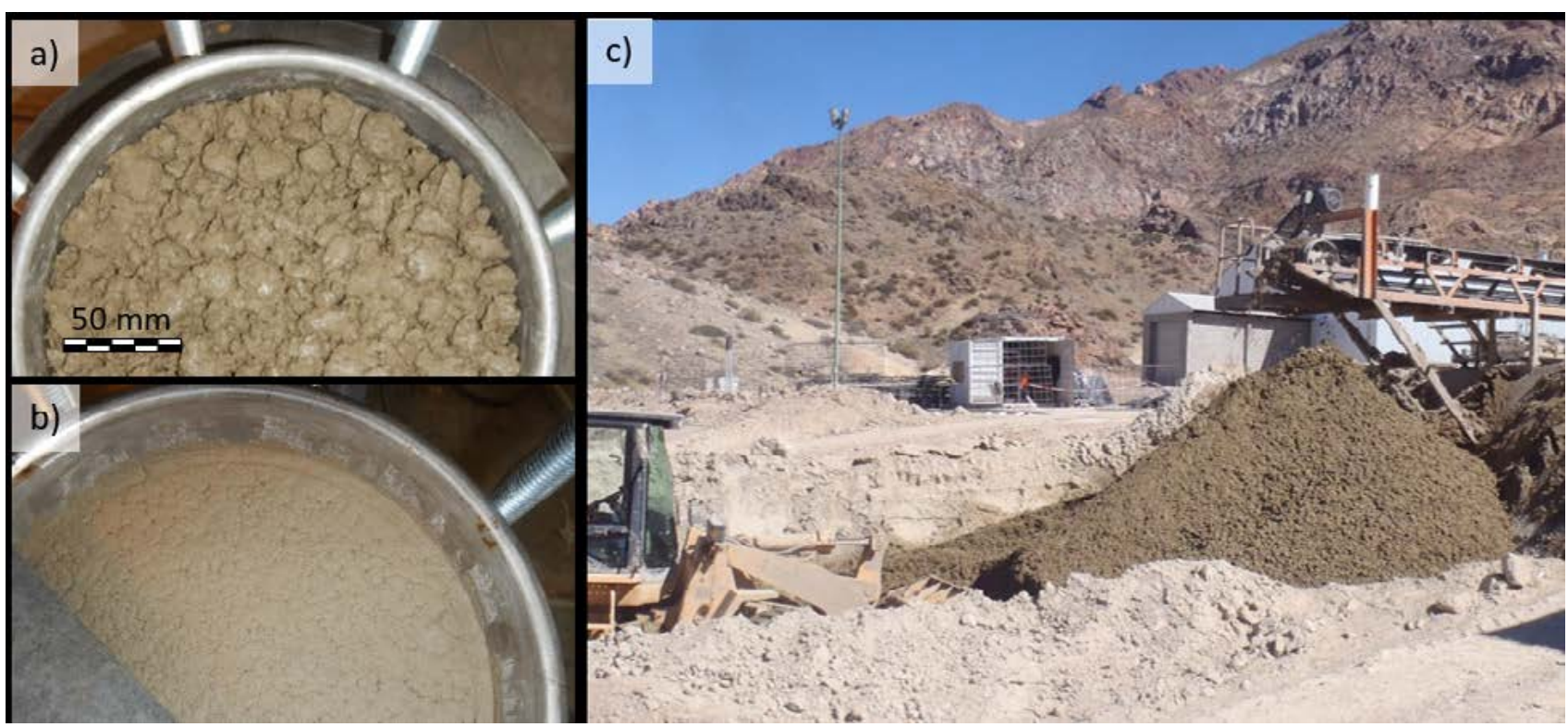

Figura 11. Tendencia de las colas $\mathrm{t} 1 \mathrm{a}$ agregarse formando grumos: a) antes del ensayo edométrico, b) después del ensayo edométrico y c) en stock-pile a la salida de la planta.

Figure 11. 11 tailings material tendency to aggregate forming lumps: a) before the oedometer test, b) after the oedometer test, and c) at the stockpile at the exit of the plant.

\begin{tabular}{|c|c|c|c|c|c|c|c|c|c|c|c|}
\hline \multirow{2}{*}{ Material } & \multirow{2}{*}{ Esp. } & \multicolumn{5}{|c|}{ Condiciones iniciales de montaje } & \multicolumn{5}{|c|}{ Condiciones finales } \\
\hline & & $e$ & $\begin{array}{c}\gamma_{d} \\
{[\mathrm{kN} / \mathrm{m} 3]}\end{array}$ & $\begin{array}{c}S \\
{[\%]}\end{array}$ & $\begin{array}{c}w_{\text {inicial }} \\
{[\%]}\end{array}$ & $\begin{array}{c}w_{\text {inicial }}^{\prime} \\
{[\%]}\end{array}$ & $e$ & $\begin{array}{c}\gamma_{d} \\
{[\mathrm{kN} / \mathrm{m} 3]}\end{array}$ & $\begin{array}{c}S \\
{[\%]}\end{array}$ & $\begin{array}{c}w \\
{[\%]}\end{array}$ & $\begin{array}{c}w^{\prime} \\
{[\%]}\end{array}$ \\
\hline \multirow{4}{*}{$\mathrm{t} 1$} & 1 & 1.20 & 12.10 & 100.0 & 62.20 & 38.35 & 0.74 & 15.32 & 100.0 & 27.74 & 21.72 \\
\hline & 2 & 1.14 & 12.45 & 100.0 & 43.43 & 30.28 & 0.60 & 16.69 & 100.0 & 21.94 & 17.99 \\
\hline & 3 & 0.96 & 13.61 & 84.8 & 29.90 & 23.02 & 0.55 & 17.21 & 100.0 & 20.18 & 16.79 \\
\hline & 4 & 0.76 & 15.18 & 86.4 & 24.01 & 19.36 & 0.54 & 17.32 & 100.0 & 19.83 & 16.55 \\
\hline \multirow{3}{*}{$\mathrm{t} 2$} & 5 & 0.73 & 15.42 & 93.4 & 25.00 & 20.00 & 0.42 & 18.73 & 100.0 & 15.56 & 13.46 \\
\hline & 6 & 0.89 & 14.07 & 45.6 & 15.00 & 13.04 & 0.58 & 16.85 & 69.0 & 15.00 & 13.04 \\
\hline & 7 & 1.51 & 10.60 & 17.9 & 10.00 & 9.09 & 0.68 & 15.91 & 40.0 & 10.00 & 9.09 \\
\hline
\end{tabular}

Tabla 4. Condiciones iniciales y finales de los especímenes ensayados en edómetro (e: índice de poros, $\mathrm{g}_{d}$ : peso unitario seco, $S$ : grado de saturación, $w$ : humedad, $w^{\prime}$ : fracción másica de agua).

Table 4. Initial and final conditions of the specimens tested in the oedometer (e: void ratio, gd: dry unit weight, S: degree of saturation, w: humidity, w': mass fraction of water).

betas consolidaron bajo peso propio, expulsando el agua segregada. Por lo tanto, al aplicar el primer incremento de carga, las probetas 1 y 2 tenían humedades de 37 y $39 \%$ respectivamente e índices de poros cercanos a 1. Consistentemente, las líneas de compresión de ambos especímenes son casi idénticas. En el depósito ocasionalmente se han observado consistencias fluidas como estas, normalmente como resultado de problemas de operación de los filtros. Esto no ha significado un inconveniente para la operación del depósito, ya que el agua en exceso puede drenar a través de las capas de estéril. En los especímenes preparados a humedades menores no se observó segregación.

Las compresibilidades de los materiales $\mathrm{t} 1$ y $\mathrm{t} 2$ resultan similares en el rango de tensiones aplicadas en el ensayo edométrico, aun cuando las humedades son muy diferentes. Esto puede atribuirse a la acción del agente floculante utilizado en el proceso metalúrgico en el material t1, que le confiere una mayor capa- 
cidad de retención de agua. Las líneas de compresión obtenidas para el material t1 muestran un efecto de preconsolidación para humedades de preparación decrecientes. La tensión de preconsolidación está entre 50 y $100 \mathrm{kPa}$. Sin embargo, la línea de compresión virgen no resulta única para todos los especímenes, sino que se nota un efecto de la condición de montaje. Los especímenes preparados con consistencia inicial fluida (E1 y E2) tienen sus líneas de compresión virgen desplazadas entre 0.05 y 0.1 unidades de índice de poros por arriba de las obtenidas con los especímenes preparados a humedades menores (E3 y E4). En contraste, las curvas de compresión virgen del material t2, no alcanzan a reunirse bajo el rango de tensiones aplicado. En otras palabras, el efecto de la condición de montaje no alcanza a borrarse, aunque el nivel de tensiones máximo aplicado es considerablemente elevado (1500 kPa). Si bien las granulometrías de los dos materiales ensayados difieren, ambos clasifican como limo arenoso (ML-SM) y por lo tanto se puede suponer que las notables diferencias en el comportamiento mecánico se deben a la acción del agente floculante.

La Figura 12b muestra la evolución del grado de saturación en los ensayos edométricos en función de la tensión vertical aplicada. El grado de saturación se calculó con índice de poros al final del periodo de espera de cada incremento de carga, la humedad del material en ese momento y el peso específico de los sólidos. Para ello se hizo la hipótesis que la humedad es constante e igual a la inicial mientras la probeta no alcanza la saturación (el agua es retenida por capilaridad). Una vez que se alcanza la saturación, puesto que la carga y la deformación crecen monotónicamente, se supone que el ensayo continúa saturado hasta el final. Esto pudo corroborarse con la observación de que la expulsión de agua comenzaba aproximadamente en el mismo momento en que se calculaba un grado de saturación 1 para las probetas que alcanzaron esa condición durante el ensayo. Las probetas 1 y 2 , inicialmente saturadas, se mantienen saturadas durante todo el ensayo. Las probetas $3,4 \mathrm{y}$ 5 , en cambio, parten de una condición no saturada y alcanzan la saturación en algún punto del proceso de carga. A menor humedad inicial, mayor es la tensión vertical que se debe aplicar para provocar la saturación. Las probetas 6 y 7 no alcanzan la saturación bajo la máxima tensión aplicada, aunque los datos sugieren que podrían hacerlo a tensiones mayores.

Para los objetivos de este trabajo resulta útil presentar los mismos datos de los ensayos edométricos, en forma de un gráfico de humedad vs. tensión vertical (Figura 13a). Esto se hace solamente para el material t1, que son las colas almacenadas en el depósito. Los especímenes 1 y 2 , saturados desde el inicio del ensayo, reducen su humedad monotónicamente con el aumento de la tensión vertical aplicada, ya que a lo largo de todo el proceso de carga expulsan un volumen de agua idéntico al cambio de volumen de la probeta. Las probetas 3 y 4 , en una primera etapa expulsan aire, reduciendo su volumen a humedad constante. Pero a un cierto nivel de deformación los poros se saturan y a partir de allí la probeta comienza a expulsar agua, por lo que la humedad comienza a disminuir. Idealmente, las trayectorias $\log (\sigma)-w$ en compresión deberían converger en una línea única, que definiría

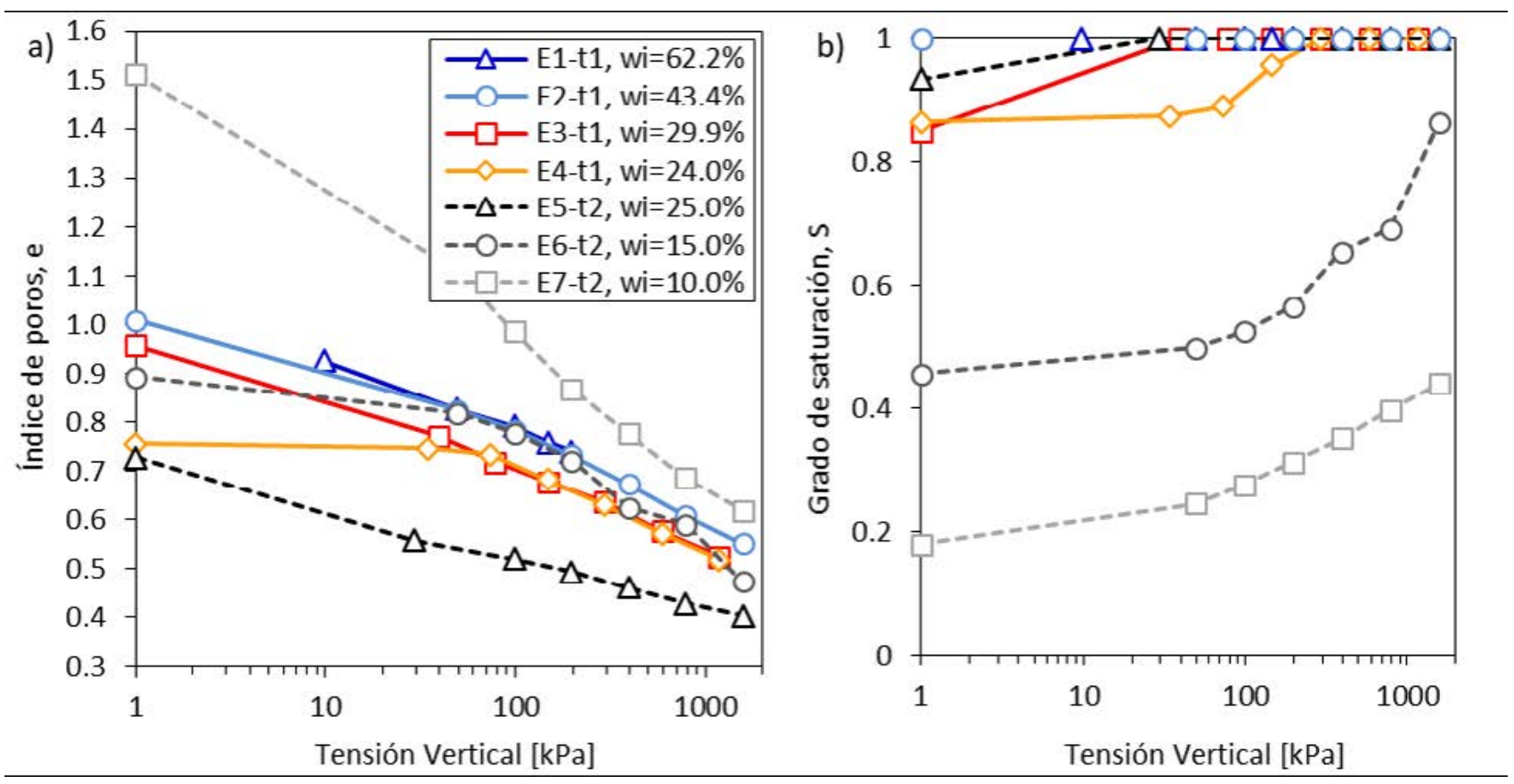

Figura 12. Ensayos edométricos: a) Curvas de compresión virgen y b) Evolución del grado de saturación. Figure 12. Oedometer tests: a) virgin compression lines and b) evolution of the degree of saturation. 
la combinación de valores de humedad y tensión que provoca la saturación del material. A la vez esa línea de saturación conformaría una frontera que no se puede traspasar, de la misma manera que la línea de compresión virgen lo es en el plano de compresión.

El concepto de una línea de saturación es útil ya que, conocida la tensión vertical a que va a ser sometido el material (en función de la tapada), se puede deducir de ella el valor de humedad objetivo, $w_{0}$ (Figura 2), que asegure que las colas no se saturarán. Sin embargo, los datos experimentales disponibles muestran que los puntos de humedad de saturación no convergen en una línea única. El comportamiento observado sugiere que existe una influencia de las condiciones de elaboración de las probetas, que no se elimina con el aumento de la carga (Figuras 11a y $11 \mathrm{~b}$ ). de laboratorio, pero con una media que queda sistemáticamente por arriba del conjunto de puntos saturados obtenidos con el edómetro. La variabilidad es aún mayor en los datos de campo y esto puede asociarse a la mayor cantidad de factores involucrados en el método de vertido utilizado en el depósito, que pueden influir en la estructura del material. En todos los casos, los datos de calicatas provienen de las colas vertidas sin arado. Se ajustó la siguiente ecuación a los datos de campo de humedad de saturación mostrados en la Figura 13a:

$$
w_{\text {sat }}=d-e \cdot \log \left(\frac{\sigma_{v}}{p_{a t m}}\right)+\epsilon_{\text {sat }}\left(0, s_{\text {sat }}\right)
$$

donde es el tensión vertical (vertical stress) y $d$ y $e$ son parámetros de ajuste (fitting parameters) y $p_{\text {atm }}$ es la presión atmosférica (atmospheric pressure) inclui-

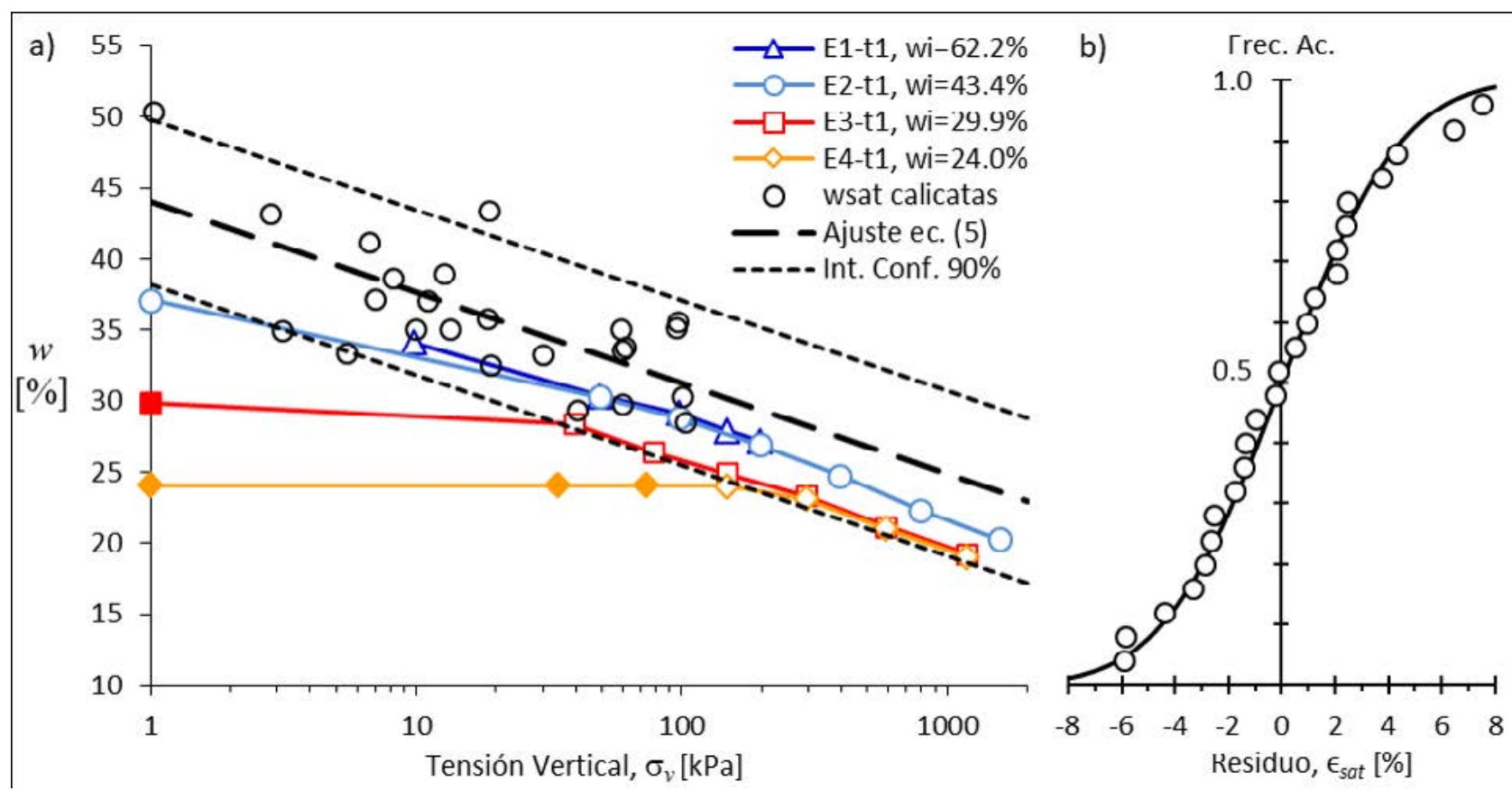

Figura 13. a) Evolución de la humedad en ensayos edométricos. Los puntos llenos corresponden a estados no saturados del material mientras que los sin relleno corresponden a estados saturados. Datos de humedad de saturación de campo deducidos de mediciones de peso unitario en calicatas y la tapada, ajustados con Ecuación (5) y b) Análisis de varianza del residuo del ajuste de la Ecuación (5).

Figure 13. a) Evolution of water content in oedometer tests. Filled markers correspond to unsaturated states of the specimen while open markers correspond to saturated states. Field saturation water content values deduced from unit weight measurements in trenches and cover depths fitted with equation (5) and b) variance analysis of residual from adjustment of equation (5).

Para corroborar y complementar los datos de laboratorio, se incluyen en la Figura 13a datos de campo de humedad de saturación vs. tensión vertical obtenidos de mediciones en calicatas efectuadas en diferentes puntos y momentos de construcción del depósito de colas filtradas de Casposo. La humedad de saturación se calculó a partir de mediciones in situ del peso unitario (ASTM D1556) y la determinación en laboratorio de la densidad de sólidos (ASTM D854-14). La tensión vertical se estimó en cada caso con la tapada, el perfil de materiales excavados y su peso unitario promedio. La nube de puntos resultantes está cerca de las determinaciones da para normalizar la tensión, de manera que los parámetros de la Ecuación (5) resulten adimensionales. Los dos primeros sumandos dan el valor esperado de la humedad de saturación, $\overline{\mathrm{w}}_{\text {sat' }}$ para una cierta tensión vertical, $\sigma_{v} \cdot \epsilon_{\text {sat }}$ es el residuo, es decir la diferencia entre las mediciones de campo y el valor esperado. Se asume que el residuo es una variable aleatoria normalmente distribuida, con media nula y desviación estándar, $s_{s a t^{*}}$ Los parámetros de la Ecuación (5) se calcularon mediante regresión con el criterio de mínimos cuadrados y los valores obtenidos se indican en la Tabla 5. 
El resultado del ajuste de la Ecuación (5) a los datos de las calicatas se muestra en la Figura 13a como una recta de valores esperados (línea de trazos gruesos) y los límites del intervalo de confianza del $90 \%$ (líneas de trazos finos). En la Figura 13b se muestra el análisis de varianza del residuo del ajuste. El uso de la Ecuación (5) implica una extrapolación, ya que es imposible obtener datos de campo a mayores profundidades que el rango indicado en la Tabla 5. Sin embargo, ofrece una cierta confianza el hecho que el ajuste de la línea saturación resulta esencialmente paralelo a las líneas de saturación obtenidas en laboratorio y que los datos de laboratorio caen dentro del intervalo de confianza del $90 \%$ obtenido con los datos de campo. El análisis presentado se limita a las colas vertidas sin arado. No se dispone de datos de campo de humedad de saturación para el método de vertido con arado.

\begin{tabular}{cccccc}
$\begin{array}{c}\text { Método de } \\
\text { vertido }\end{array}$ & Periodo $\begin{array}{c}\text { Rango de prof. de las } \\
\text { determinaciones } \\
\text { de peso } \\
\text { unitario } \\
{[\mathrm{m}]}\end{array}$ & \multicolumn{3}{c}{$\begin{array}{c}\text { Parametros } \\
\text { de ajuste de la }\end{array}$} \\
\hline Sin arado & 2011 a 2018 & $0.00-7.00$ & 0.314 & 0.064 & 0.035 \\
\hline
\end{tabular}

Tabla 5. Condiciones de obtención de datos de campo y parámetros de ajuste de la Ecuación (5).

Table 5. Tailings sampling conditions and fitting parameters for equation (5).

\section{Discusión}

La comparación de la humedad resultante del proceso de secado, $w_{0}$, con la humedad de saturación, $w_{\text {sat }}$ , permite evaluar el grado de saturación que se alcanzará al momento del cierre $S_{c}$, estimada la tensión vertical que recibirá la capa de colas analizada. Esto a su vez permite poner a punto el procedimiento de vertido, por ejemplo seleccionando el tiempo de permanencia adecuado para lograr una humedad objetivo, $w_{o}$, que asegure una condición de cierre satisfactoria.

Los datos experimentales presentados en las secciones anteriores muestran que, definido un tiempo de secado $t$ y un valor de tensión vertical, $\sigma_{v}$, que actúa en una capa a una cierta profundidad, ambos parámetros, $w_{0}$ y $w_{\text {sat }}$, son variables aleatorias. El análisis realizado justifica, al menos en el caso estudiado, que ambas variables se pueden describir mediante una distribución normal en torno a un valor medio dado por expresiones matemáticas relativamente sencillas, que pueden ajustarse con el criterio de mínimos cuadrados.

En la discusión que sigue, se asume que las variables $w_{0}$ y $w_{\text {sat }}$ son independientes. Esta hipótesis se puede justificar solo parcialmente. En principio, los factores que determinan $w_{0}$ son esencialmente meteorológicos y climatológicos, mientras que $w_{\text {sat }}$ depende del comportamiento mecánico de las colas durante la etapa 3. Si bien existe (se ha mencionado en la sección 4) una posible influencia de las condiciones de vertido (básicamente de la humedad) en la estructura y compresibilidad del material resultante $y$, evidentemente, ese mismo factor afecta el resultado del proceso de secado, de momento no se dispone de una base de datos adecuada para poder analizar su efecto en la eventual dependencia entre $w_{o}$ y $w_{\text {sat }}$.

Aceptando la hipótesis de independencia de $w_{0} y$ $w_{\text {sat }}$, es posible calcular la probabilidad que $w_{0} \geq w_{\text {sat }}$, que es igual a la probabilidad que el material alcance la saturación al final de la construcción:

$P\left(w_{0} \geq w_{\text {sat }}\right)=P\left(w_{0}-w_{\text {sat }} \geq 0\right)=P\left(S_{c} \geq 100 \%\right)$

La Ecuación (6) se puede extender a cualquier grado de saturación, $S$, reemplazando $w_{\text {sat }}$ por $S . w_{\text {sat }}$ La presión vertical se puede determinar a partir de la tapada $T$, asumiendo un valor medio del peso unitario seco y de la humedad del material de las capas superiores $\left(14.6 \mathrm{kN} / \mathrm{m}^{3}\right.$ y $23 \%$ para este análisis), $\sigma_{v}\left[\mathrm{kN} \mathrm{m}^{2}\right.$ ]$=T[m]^{*}[14.6(1+0.23)]$ y calcular $w_{\text {sat }}$ para una capa a una cierta profundidad. A partir de la Ecuación (6) se puede escribir:

(7)

$P\left(S_{c} \geq S\right)=P\left(w_{0}-S \cdot w_{s a t}(T) \geq 0\right)=P(X \geq 0)$

Donde $X=w_{0}-S . w_{\text {sat }}$ es también una variable aleatoria con valor medio $\bar{X}=\overline{\mathrm{w}}_{0}-S . \overline{\mathrm{w}}_{\mathrm{sat}}$ y desviación estándar: $s_{x}=\sqrt{ }\left(s_{0}{ }^{2}+\left(S . s_{\text {sat }}\right)^{2} y \bar{w}_{0} y \bar{w}_{\text {sat }}\right.$ son los valores medios dados por los dos primeros sumandos de las ecuaciones (3) y (5) respectivamente.

Utilizando las ecuaciones (3), (4), (5) y (7) para una capa de colas que se sometió a un periodo de secado de 45 días sin arado (Fig. 9), se obtienen las curvas mostradas en la Figura 14, de donde puede leerse, para una capa que tendrá una cierta tapada al final de la construcción, la probabilidad de alcanzar diferentes valores de grado de saturación al momento del cierre del depósito. Estrictamente hablando, ese grado de saturación es el correspondiente a la profundidad de referencia, en este caso $50 \mathrm{~cm}$, es decir a media altura de la capa de colas vertidas sin arado. Sin embargo, si se acepta que las medidas de humedad a la profundidad de referencia son un buen estimador de la humedad promedio en la altura de la capa, el grado de saturación al que hace referencia la Figura 14 puede interpretarse como un valor promedio en la altura de la capa.

Lógicamente, los valores de grado de saturación mayores al $100 \%$ indicados en la Figura 14 no son físicamente posibles. En realidad, indican el exceso de agua que eventualmente se expulsará cuando la tapada alcance el valor supuesto. Por ejemplo, en una capa con una tapada de 20 metros, un $15 \%$ del volumen de las colas alcanzará la saturación. El exceso de agua generado en esos puntos podrá redistribuirse en la misma capa de colas o drenar hacia las capas de estéril subyacente, por lo que no significa un problema para la estabilidad y la operatividad del depósito. 
Utilizando las curvas de la Figura 14, un proyectista podría establecer un criterio de diseño para el proceso. Por ejemplo, si dispone de un periodo de secado de 45 días (determinado por la producción de la planta y la geometría del depósito), podría requerir un grado de saturación máximo del $90 \%$ con una probabilidad de excedencia del $20 \%$. Para cumplir ese requisito, las curvas de la Figura 14 indican que la tapada no debería superar los $10 \mathrm{~m}$. Por lo tanto, resultaría un criterio bastante restrictivo. Si quisiera ampliar el rango de trabajo a tapadas mayores, debería tomar otras medidas como aumentar el tiempo de secado (aunque ya vimos que en este caso un mayor tiempo de permanencia en Etapa 2 no produce mejoras significativas en el secado), modificar el proceso de filtrado para lograr humedades de vertido menores o incorporar algún manejo posterior al vertido (arado) que permita intensificar el secado.

Sin embargo, para el caso particular estudiado, es interesante notar que, por efecto de barrera capilar, el agua contenida en las colas se retendrá dentro de la misma capa, salvo en aquellos puntos en donde se haya alcanzado la saturación porque allí se romperá la barrera (Oldecop et al., 2017). En esas condiciones y considerando la distribución de grados de saturación mostrada por cualquiera de las curvas de la Figura 14 , es esperable que dentro de cada capa ocurra una redistribución de humedad por flujo lateral, desde las zonas con mayor grado de saturación (mayor potencial) a las zonas con menor grado de saturación (menor potencial). En el límite, y para el caso ideal de una capa que no pierde ni gana humedad, el grado de saturación de la capa debería tender al valor con probabilidad de excedencia del $50 \%$. La Figura 14 indica entonces que el grado de saturación a largo plazo tendería a ubicarse dentro de un rango entre 67 y $90 \%$, para tapadas entre 2 y 50 metros. Esta condición final es bastante aceptable y justificaría utilizar como criterio de diseño un valor de grado de saturación admisible (por ejemplo, el 90\%) con una probabilidad de excedencia del $50 \%$, lo que en este caso significaría que se podrían admitir tapadas de hasta 50 metros. Una evidencia de que existe flujo lateral dentro de las capas de colas se muestra en la Figura 15, en donde puede verse un bandeado de diferente coloración en los taludes del depósito. Es el efecto de la precipitación de sales por evaporación en correspondencia con las capas de colas expuestas a la interacción con la atmósfera. Esta condición de frontera de interacción con la atmósfera produce un gradiente de potencial dentro de las capas, induciendo un flujo lateral hacia la frontera. Esta pérdida de agua no se ha tenido en cuenta en el presente análisis, pero en todo caso es un mecanismo adicional de pérdida de agua y por lo tanto favorable para la condición final de las colas.

Finalmente, pueden hacerse algunas consideraciones sobre el diseño de este tipo de depósitos. EI tiempo de secado necesario en Etapa 2, condiciona geométricamente el depósito ya que determina el área de trabajo necesaria para poder acomodar la pro-

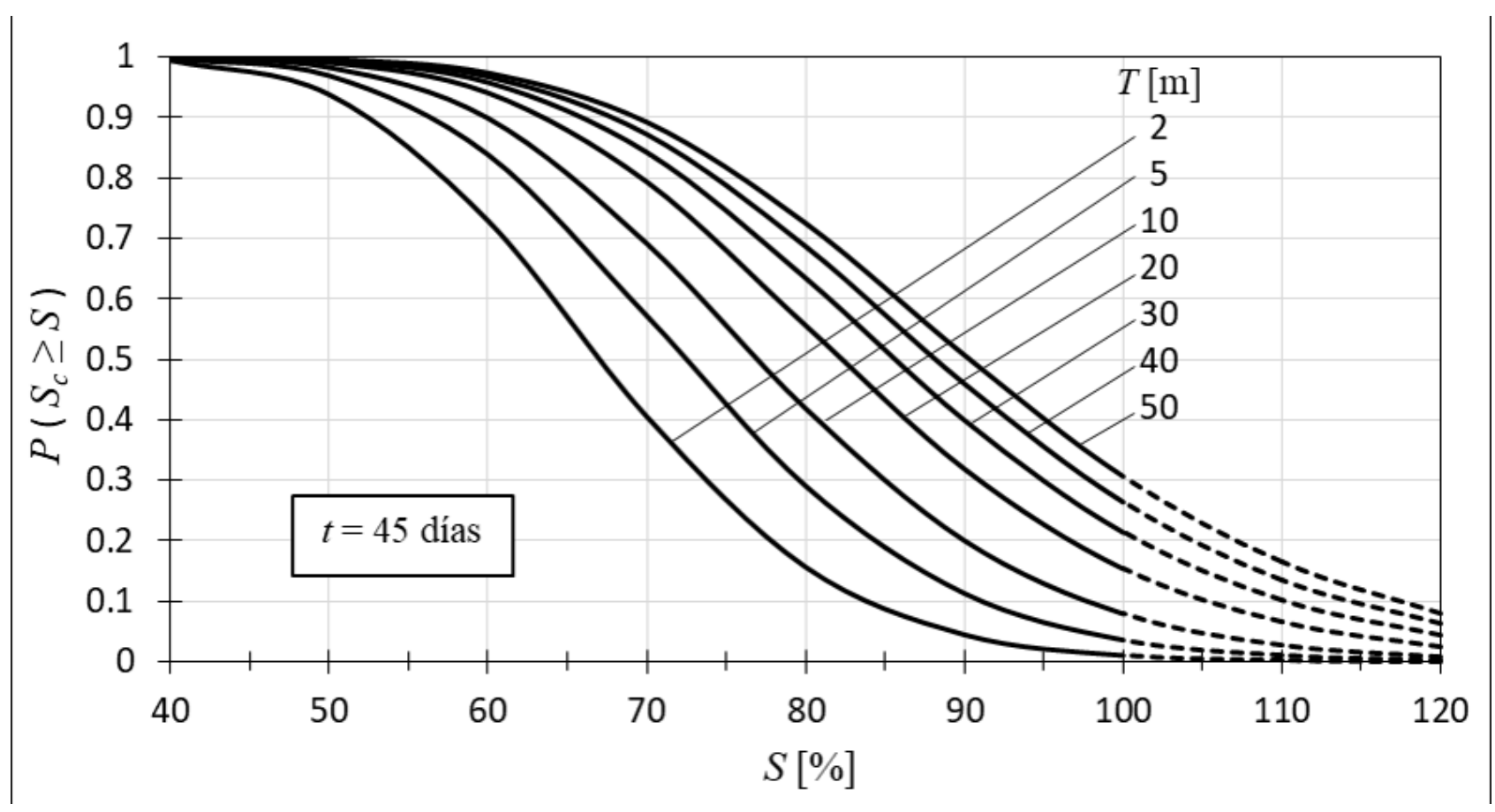

Figura 14. Probabilidad de excedencia de un valor de grado de saturación, $S$ (valor promedio en el espesor de la capa), al finalizar la construcción del depósito, en una capa de colas secadas durante 45 días sin arado, para distintas profundidades de la tapada.

Figure 14. Exceedance probability of a degree of saturation value, $S$ (average value the layer height), at the closure stage, in a tailings layer dried during a 45-day period with no ploughing, for different cover depths. 
ducción de colas. Debido a la necesidad de introducir taludes estables y constructivamente viables, el área tenderá a reducirse a medida que el depósito crece. Sin embargo, una propiedad interesante que surge del análisis presentado es que los requerimientos de secado son menos rigurosos a medida que disminuye la tapada final, por lo que las capas superiores del depósito requerirán menos tiempo de permanencia en Etapa 2, lo que relaja el requisito de área de trabajo. Un efecto inverso puede presentarse en el caso de depósitos que rellenan un valle, haciendo que las primeras etapas de llenado sean críticas en relación al espacio necesario.

Como se comprende, la puesta a punto de todo el proceso resulta crucial para el éxito de la operación. Una gran dificultad para el proyectista es la escases de datos publicados sobre los dos fenómenos básicos que controlan el problema: el secado en contacto con la atmósfera y la consolidación de las colas filtradas. A la fecha, los autores no han encontrado otros trabajos similares publicados sobre el tema. En ausencia de datos de campo, el diseño del depósito y del proceso puede basarse en ensayos edométricos para caracterizar la compresibilidad de las colas y en ensayos de columna para estudiar su secado (Oldecop et al., 2017). Sin embargo, como se ha mostrado aquí, los datos de ensayo pueden diferir mucho de la realidad por lo que deben utilizarse con cautela y el diseño resultante debe contar con márgenes de redundancia amplios. Debe tenerse en cuenta que todo el proceso y posiblemente también la geome- tría del depósito deba someterse a ajustes durante los primeros estadios de la operación. Es importante entonces, en esa fase inicial, llevar a cabo un proceso sistemático de muestreo para estudiar la evolución de la humedad y la compresibilidad de las colas en las condiciones de vertido reales. Sería útil la instalación de instrumental de auscultación para medición de la compresibilidad de las colas. Obviamente, sería aún más útil contar con medición directa del grado de saturación, pero no existen a la fecha instrumentos confiables para tal fin y menos aún que resistan las condiciones químicamente agresivas dentro de las colas.

\section{Conclusiones}

Los datos presentados en el este trabajo son aún escasos para poder caracterizar por completo las colas filtradas $y$, evidentemente, son sólo válidos para el caso particular estudiado. Sin embargo, no hay en conocimiento de los autores trabajos publicados con datos similares. La contribución principal que se pretende hacer es un marco conceptual que sirva para diseñar y operar este tipo de depósitos. En el caso de depósitos de colas filtradas, el diseño no solo incluye la geometría del depósito y sus detalles constructivos sino también la operación, es decir forma de vertido, manejos post-vertido, espesor de capas de colas, etc. Ambos aspectos están íntimamente ligados y no pueden diseñarse por separado.

Como se ha mostrado con los datos experimen-

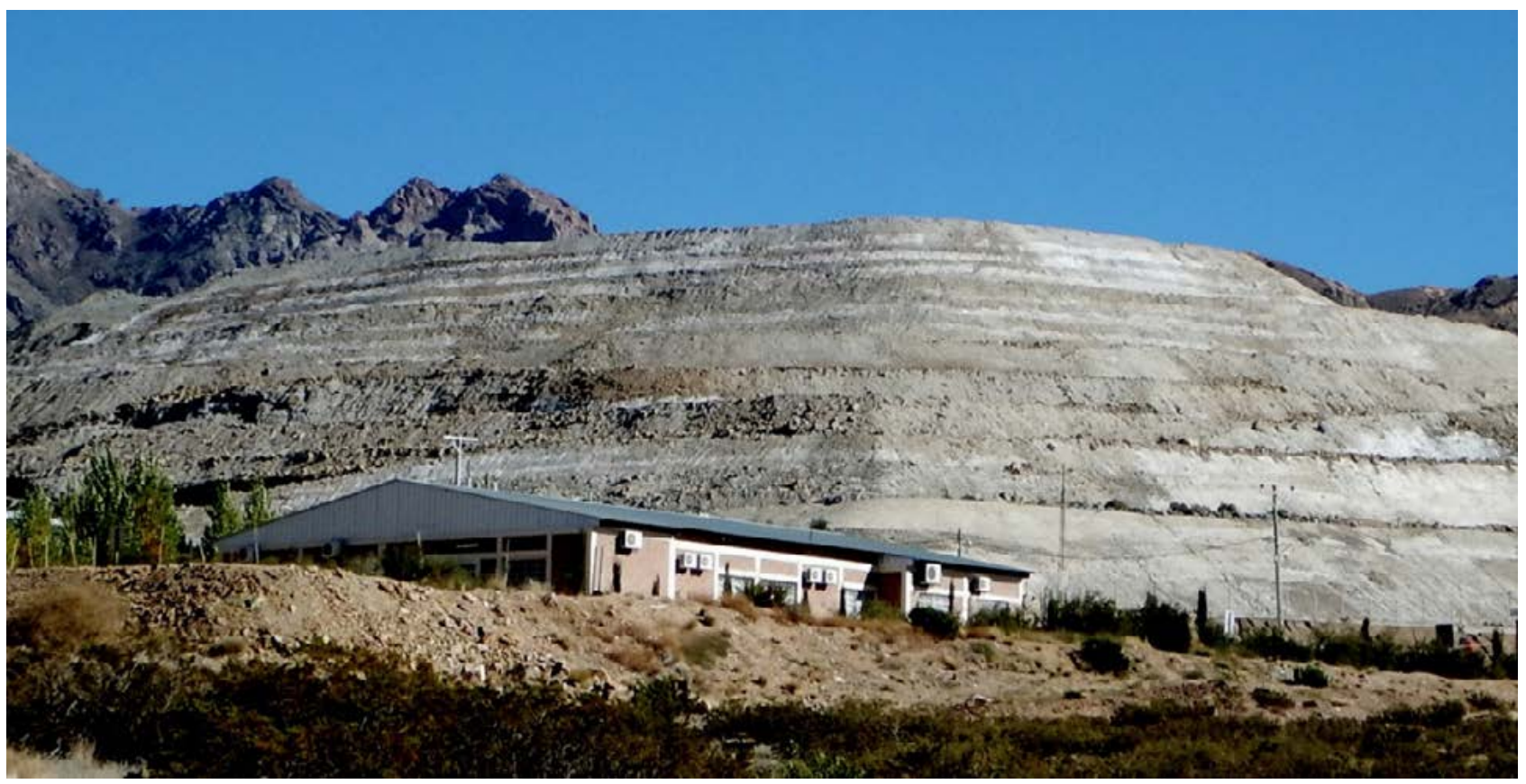

Figura 15. Bandeado claramente visible en los taludes, resultado de la precipitación de sales (eflorescencias) en los bordes de las capas de colas por evaporación del agua que prueba que existe flujo lateral de agua en las capas de colas debido al gradiente de potencial de agua creado por la atmósfera.

Figure 15. Clearly visible banding at the slopes, as the result of the salts precipitating (efflorescence) at the edges of the tailings layers as water evaporates, indicating water flowing horizontally due to the water potential gradient induced by the atmosphere. 
tales presentados, carece de sentido fijar un valor único de humedad objetivo. Esta ha sido una práctica habitual en el pasado, pero dada la variabilidad de las humedades de filtrado y de final de la Etapa 2 demostrada aquí, la mayor parte de las veces se convierte en un objetivo imposible de cumplir y por lo tanto pierde toda su utilidad como parámetro de diseño y operación. Es mucho más adecuado trabajar con rangos, valor esperado y desviación estándar o valores característicos con una cierta probabilidad de excedencia.

De la misma manera, parece difícil caracterizar en forma determinista el proceso de compresión de las colas bajo el peso de las tongadas superiores. La base de datos disponibles es muy limitada, aún para el caso estudiado, pero los datos de campo parecen indicar que es también adecuado caracterizar el comportamiento mecánico mediante un modelo de probabilidad.

El resultado del análisis presentado es también una distribución de valores de grado de saturación al final de la construcción, en lugar de un valor único deseado. Para el diseño en capas alternadas de colas y estéril adoptado en Casposo, no es un problema el que en algunos puntos se alcance localmente la saturación, ya que la presencia de las capas de estéril permite evacuar o redistribuir el agua en exceso y además evita la generación de presiones de poro que podrían afectar la estabilidad física del depósito. Por otro lado, se puede esperar que durante todo el proceso de construcción y aún después de terminado, ocurra redistribución de agua dentro de las capas, de manera que el grado de saturación tienda a uniformarse acercándose al valor medio estimado para la capa. En el caso presentado, esos valores medios resultan muy razonables de acuerdo a la práctica y experiencia internacional.

Evidentemente, un diseño ajustado del depósito sólo puede alcanzarse cuando se tienen datos de campo, ya que, como se ha mostrado, los datos de laboratorio pueden diferir mucho del comportamiento real. Esto supone un gran obstáculo para el proyecto y la única solución es recurrir a un diseño conservador, con márgenes de operación amplios (por ejemplo, áreas de trabajo mayores que las estrictamente necesarias). El diseño debe contemplar también que el proyecto tendrá una o más adecuaciones a medida que se adquiera experiencia y datos reales en las primeras fases de la operación. Para ello es importante diseñar campañas de obtención y análisis de datos que luego sirvan de base para ajustar el proyecto.

\section{Reconocimientos}

El presente trabajo se desarrolló bajo el patrocinio de Troy Resources Argentina y la coordinación de la Fundación UNSJ. Los autores agradecen el financiamiento $y$ apoyo recibidos.

\section{Referencias}

ASTM D854-14, Standard test methods for specific gravity of soil solids by water pycnometer, ASTM International, West Conshohocken, PA, 2014.

ASTM D1556 / D1556M-15e1. Standard test method for density and unit weight of soil in place by sandcone method, ASTM International, West Conshohocken, PA, 2015.

ASTM D2216-19. Standard test methods for laboratory determination of water (moisture) content of soil and rock by mass, ASTM International, West Conshohocken, PA, 2019.

Bianchi, A. and Cravero, S. 2010. Atlas climático digital de la república argentina. Instituto nacional de tecnología agropecuaria, Salta, Argentina.

Caldwel, J. Crystal, C. 2015. Filter-pressed tailings facility design, construction and operation guidelines. Tailings and mine waste 2018, Vancouver, BC, Canada, 23/12/2019 https://open.library.ubc.ca/ media/download/pdf/59368/1.0320844/5

Crystal, C., Hore, C., Ezama, I. 2018. Filter-pressed dry stacking design considerations based on practical experience. Tailings and mine waste 2018, Keystone, Colorado, USA, 209-219.

Davies, M.P. and Rice, S. 2001. An alternative to conventional tailing management - "dry stack" filtered tailings. Eighth International Conference on Tailings and Mine Waste. Fort Collins, Colorado, USA, 411-422.

Lara, J., Pornillos, E., Muñoz, H. 2013. Geotechnical-geochemical and operational considerations for the application of dry stacking tailings deposits - state-of-the-art. 16th International Seminar on Paste and Thickened Tailings, Paste 2013. Belo Horizonte, Brazil.

Lupo, J. and Hall, J. 2010. Dry stack tailings - design considerations, 14th international conference on tailings and mine waste, Vail, Colorado, USA, 327-334.

Davies, M. 2011. Filtered dry stacked tailings: the fundamentals. Tailings and Mine Waste 2011. Vancouver, British Columbia, Canada.

Garino, L. 2018. Estudio del comportamiento mecánico e hidráulico de depósitos de residuos mineros ubicados en zonas áridas. Tesis doctoral, Universidad Nacional de San Juan, Facultad de Ingeniería.

Garino, L. and Oldecop, L. 2019. Evolución de la humedad en presas de relaves mineros ubicadas en climas áridos. Caso de estudio: mina Castaño Viejo, San Juan, República Argentina. Este volumen

Oldecop, L.A., Garino, L., Muñoz, J., Rodríguez, R. and García, C. 2010. Unsaturated behaviour of mine tailings in low precipitation areas. In Alonso, E. and Gens, A. (Eds)., Unsaturated Soils, Taylor \& Francis Group, London, 1425-1430

Oldecop, L.A. and Rodari, G.J. 2017. Seismic performance of filtered tailings deposits. Second Pan-American Conference on Unsaturated Soils, Dallas, Texas,USA, 257 - 280 
Oldecop, L.A., Rodari, G.J. \& Muñoz, J.J. 2017. Atmosphere interaction and capillary barrier in filtered tailings. Geotechnical and Geological Engineering Journal 35, Issue 4, 1803-1817.

Ulrich, B. and Coffin,J. 2013. Considerations for Tailings Facility Design and Operation Using Filtered Tailings. 16th International Seminar on Paste and ThickenedTailings, Paste 2013. Belo Horizonte, Bra- zil, 201-210.

Wakeman, R. 2007. The influence of particle properties on filtration. Separation and Purification Technolo$g y, 58,234-241$.

Wang, C., Harbottle, D., Liu, Q., \& Xu, Z. 2014. Current state of fine mineral tailings treatment: A critical review on the theory and practice. Minerals Engineering, 113-131.

Recibido: julio 2020

Revisado: diciembre 2020

Aceptado: enero 2021

Publicado: diciembre 2021 
